\title{
Mitochondrial fission as a driver of stemness in tumor cells: mDIVI1 inhibits mitochondrial function, cell migration and cancer stem cell (CSC) signalling
}

\author{
Maria Peiris-Pagès' ${ }^{1,3}$, Gloria Bonuccelli' ${ }^{2,3}$, Federica Sotgia ${ }^{2,3}$ and Michael P. Lisanti ${ }^{2,3}$ \\ ${ }^{1}$ Clinical and Experimental Pharmacology Group, Cancer Research UK Manchester Institute, University of Manchester, \\ Manchester, UK \\ ${ }^{2}$ Translational Medicine, School of Environment and Life Sciences, Biomedical Research Centre (BRC), University of Salford, \\ Greater Manchester, UK \\ ${ }^{3}$ The Paterson Institute, University of Manchester, Withington, UK \\ Correspondence to: Federica Sotgia, email: fsotgia@gmail.com \\ Michael P. Lisanti, email: michaelp.lisanti@gmail.com \\ Keywords: mitochondrial fission; OXPHOS; cancer stem-like cells (CSCs); cell migration; metastasis \\ Received: December 12, $2017 \quad$ Accepted: January 09, $2018 \quad$ Published: January 19, 2018 \\ Copyright: Peiris-Pagès et al. This is an open-access article distributed under the terms of the Creative Commons Attribution Li- \\ cense 3.0 (CC BY 3.0), which permits unrestricted use, distribution, and reproduction in any medium, provided the original author \\ and source are credited.
}

\section{ABSTRACT}

Mitochondria are dynamic organelles frequently undergoing fission and fusion events to maintain their integrity, bioenergetics and spatial distribution, which is fundamental to the processes of cell survival. Disruption in mitochondrial dynamics plays a role in cancer. Therefore, proteins involved in regulating mitochondrial dynamics are potential targets for treatment. mDIVI1 is an inhibitor of the mitochondrial fission protein DRP1, which induces i) mitochondrial oxidative stress and ii) effectively reduces mitochondrial metabolism. We show here that mDIVI1 is able to inhibit 3D tumorsphere forming capacity, cell migration and stemness-related signalling in breast cancer cells, indicating that MDIVI1 can potentially be used for the therapeutic elimination of cancer stem cells (CSCs).

\section{INTRODUCTION}

Mitochondria are extremely dynamic organelles in constant division, elongation and connection to each other to form tubular networks or fragmented granules in order to satisfy the requirements of the cell and adapt to the cellular microenvironment. The balance of mitochondrial fusion and fission dictates the morphology, abundance, function and spatial distribution of mitochondria, therefore influencing a plethora of mitochondrial-dependent vital biological processes such as ATP production, mitophagy, apoptosis and calcium homeostasis [1]. In turn, mitochondrial dynamics can be regulated by mitochondrial metabolism, respiration and oxidative stress [2]. Thus, it is not surprising that an imbalance of fission and fusion activities has a negative impact on several pathological conditions, including cancer. Cancer cells often exhibit fragmented mitochondria, and enhanced fission or reduced fusion is often associated with cancer, although a comprehensive mechanistic understanding on how mitochondrial dynamics affects tumorigenesis is still needed [3].

A master regulator of the mitochondrial fission machinery is the cytoplasmic dynamin-related protein 1 (DRP1, also known as DNM1L). During mitochondrial fission DRP1 is recruited to the mitochondria, where it interacts with its outer mitochondrial membrane receptors. Subsequently, DRP1 polimerizes and promotes mitochondrial constriction and fission, activities that are fuelled by its GTPase activity [4].

The discovery of a derivative of quinazolinone, the mitochondrial DIVision Inhibitor 1 (mDIVI1), a small molecule that selectively and reversibly inhibits DRP1 [5] (Figure 1A), has helped to shed new light on the role of mitochondrial dynamics in cancer. mDIVI1 has been shown to target DRP1 by binding and suppressing both the 
DRP1 self-assembly into ring-like structures around the mitochondria and its capacity to catalyze GTP hydrolysis. mDIVI1 prompts a rapid formation of interconnected mitochondria without overtly affecting other cellular structures such as the cytoskeleton or the endoplasmic reticulum. The IC50 of mDIVI1 ranges from 1 to 50 $\mu \mathrm{M}$ depending on the cell type [5]. In contrast to the cytoprotective effect in neurons and cardiovascular cells, mDIVI1 has a cytotoxic effect in hyperproliferative cancer cells and immortalised cell lines [4]. Indeed, high DRP1 expression or activation has been described in several malignancies, and it promotes mitochondrial fission in cancer cells, which plays an important role in their proliferation and metastatic capacity [4]. Reversal of that mitochondrial fission via DRP1 inhibition with mDIVI1 induces apoptosis via cytochrome c release and cell cycle arrest by impairing the assembly of mitotic spindles and cytokinesis, consequently leading to aneuploidy [3, 4].

Finally, emerging evidence points toward a role for mitochondrial fusion and fission, and in particular for DRP1, in regulating the proliferation and survival of cancer stem cells (CSC), which are thought to be responsible for treatment failure and metastatic dissemination. DRP1-dependent fission confers chemoresistance, as chemoresistant cancer cells are prone to form highly interconnected mitochondrial networks. mDIVI1 treatment reverses this phenotype by re-sensitising chemoresistant cancer cells [6]. Moreover, high DRP1 expression and mitochondrial fragmentation contribute to maintenance of brain tumour-initiating cells, and genetic ablation of DRP1 or its pharmacological inhibition with mDIVI1 reduces their tumorigenicity in vitro and in vivo [7]. Of note, DRP1-dependent fission has been found to be essential for stem cell maintenance in immortalised mammary epithelial stem-like cells. Upon asymmetric cell division, stem-like cells contained a higher abundance of newly generated mitochondria, whereas cells with more aged mitochondria were growing less efficiently in anchorage-independent conditions and were primed to differentiate. DRP1 inhibition with mDIVI1 abolished the mitochondrial asymmetric distribution of mitochondria reducing stem-cell properties in vitro, suggesting that mitochondrial fitness regulates stemness [8]. Finally, inactivation of DRP1 with mDIVI1 seems to impede pluripotency reprogramming [9, 10]. Specific eradication of CSCs represents one of the most challenging goals of current cancer research, as it could potentially achieve a permanent cure for cancer patients.

Emerging evidence has recently shown that CSCs are critically dependant on mitochondrial function for their successful propagation and survival [11-13]. In the current study, we hypothesised that the pharmacological inhibition of DRP1-induced mitochondrial fission with mDIVI1 would target breast CSC survival via induction of mitochondrial dysfunction and repression of mitochondrial metabolism, extending our previous studies. We show that exposure of MCF7 breast cancer cells to mDIVI1 transforms these cells in metabolically less active cells, with minor ATP demand, possibly via induction of mitochondrial reactive species. These mDIVI1-treated metabolically-repressed MCF7 cells show reduced tumorsphere forming capacity, decreased migration and inhibited stemness-related signalling. mDIVI1 is also able to decrease tumorsphere formation efficiency in melanoma and lung cancer cell lines, strongly suggesting that mDIVI1 treatment decreases the abundance of CSC also in these cancers, hence pointing it out as a new antiCSC drug for cancer therapy.

\section{RESULTS}

In this study we aimed to assess the effects of mDIVI1, an inhibitor of the mitochondrial division protein DRP1, on mitochondrial function and CSC behaviour.

\section{MDIVI1 treatment reduces MCF7 cell viability}

First of all, we sought to evaluate whether mDIVI1induced inhibition of DRP1 would have a repercussion on the viability of MCF7 cells. Exposure to mDIVI1 for 48 hours did not decrease viability of MCF7 cells at a concentration of $10 \mu \mathrm{M}$, and significantly reduced it by $20 \%$ at $50 \mu \mathrm{M}$ and $100 \mu \mathrm{M}$, as measured using the PrestoBlue assay, a resazurin-based viability assay (Figure 1B). Similar results were obtained using the Bradford assay, which measures protein content, as an indicator of cell viability (Figure 1C). Only exposure to higher concentrations of mDIVI1 $(20$ and $50 \mu \mathrm{M})$ significantly reduced MCF7 protein content after 2 days of treatment. Five days of treatment did have a bigger impact on MCF7 cell viability, significantly diminishing it by $20 \%$ at a concentration of $10 \mu \mathrm{M}$, by over $65 \%$ at $50 \mu \mathrm{M}$ and $85 \%$ at $100 \mu \mathrm{M}$ (Figure 1B).

Thus, mDIVI1 reduces the viability of MCF7 cells mostly at higher concentrations and after 5 days of treatment.

\section{MDIVI1 increases MCF7 mitochondrial mass and mitochondrial oxidative stress}

In order to assess whether the inhibition of DRP1 was actually being translated into a reduction in mitochondrial fission, we aimed to stain the mitochondria of MCF7 cells with Mitotracker Deep Red, which is a marker of mitochondrial mass. mDIVI1 treatment led to more interconnected mitochondria, with slightly increased mitochondrial calibres, which could be a result of the absence of fission activity (Supplementary Figure 1). Mitochondrial mass was also quantified by flow cytometry. Mitotracker Deep Red mean fluorescence intensity was found to be significantly higher after 5 days of treatment with $50 \mu \mathrm{M}$ mDIVI1, indicating that at higher concentrations, 
mDIVI1-mediated DRP1 inhibition is able to increase the mitochondrial mass of MCF7 cells (Figure 2A).

We hypothesised that an inhibition of the mitochondrial fission would have an impact on other mitochondrial processes such as mitochondrial metabolism and general and mitochondrial oxidative stress. To test that, MCF7 cells were stained with MitoSOX and CM-
H2DCFDA, and mitochondrial superoxide and total ROS were quantified by flow cytometry. MitoSOX staining quantification in MCF7 cells revealed that exposure to both concentrations of mDIVI1 significantly increased mitochondrial superoxide production compared to vehicletreated cells (Figure 2B). However, general oxidative stress levels did not change after exposure to mDIVI1.

A<smiles>COc1cc(-n2c(S)nc3ccccc3c2=O)c(Cl)cc1Cl</smiles>

B MCF7 cells

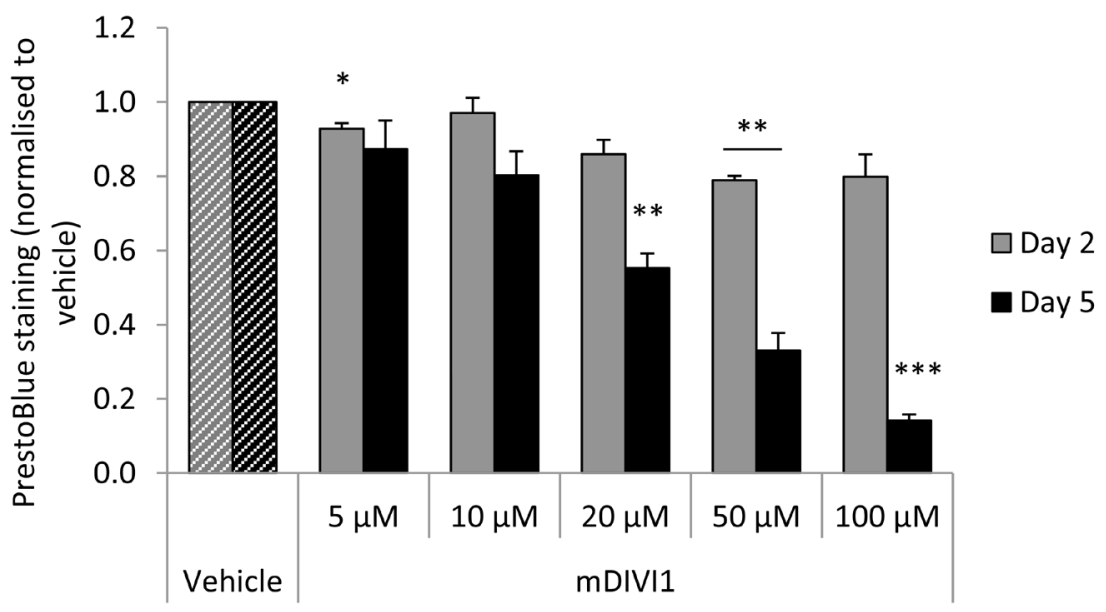

C

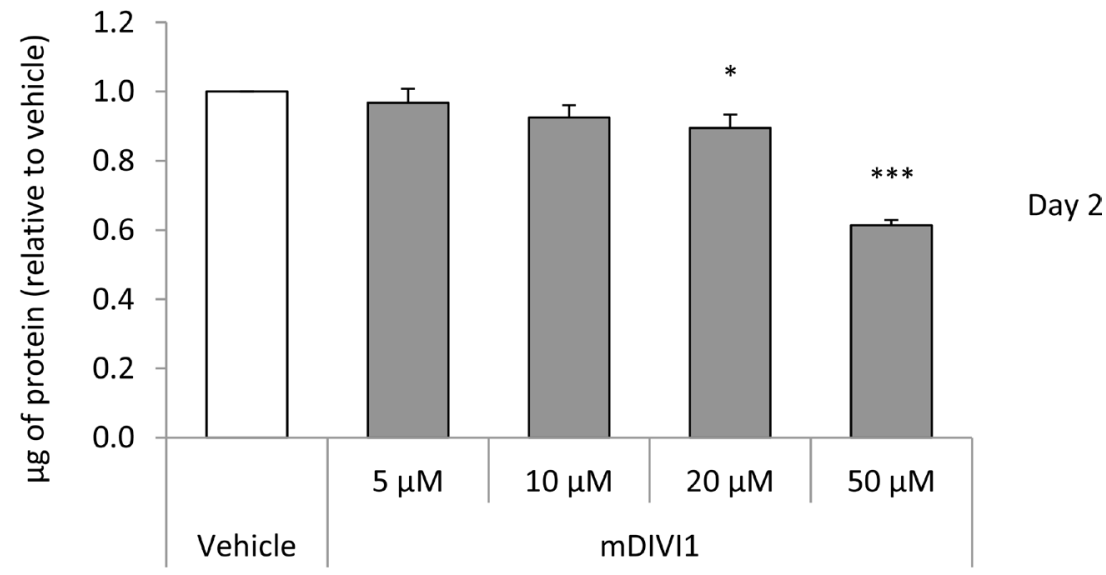

Figure 1. DRP1 inhibition by mDIVI1 reduces MCF7 cell viability. (A) mDIVI1 chemical structure (B) The mitochondrial fission inhibitor mDIVI1 did not decrease viability of MCF7 cells at a concentration of $10 \mu \mathrm{M}$, and significantly reduced it at $50 \mu \mathrm{M}$ and $100 \mu \mathrm{M}$ at 48 hours. Five days of treatment induced a bigger reduction of MCF7 viability $(N=3)$. (C) mDIVI1 slightly decreased protein content of MCF7 cells at a concentration of $10 \mu \mathrm{M}$ and reduced it by almost $40 \%$ at $50 \mu \mathrm{M}$ after 48 hours of treatment as measured using the Bradford assay $(N=6)$. Data represented as MEAN \pm SEM. One sample $t$ test, ${ }^{*} p$ value $<0.05,{ }^{* *} p$ value $<0.01$ and ${ }^{* * *} p$ value $<0.001$ 
Only 5 days of treatment showed a slight trend toward an increase in the production of total ROS (Figure 2C). Of note, whereas the increase in general ROS goes in line with the increase in mitochondrial content, the boost in the levels of mitochondrial superoxide in mDIV1treated cells is actually bigger than the observed increased mitochondrial content.

Thus, mDIVI1 treatment slightly increase mitochondrial mass and clearly induced the generation of mitochondrial superoxide without any major effects on MCF7 general oxidative stress.

\section{MDIVI1 reduces glycolytic capacity, respiration and ATP production of MCF7 cells}

We hypothesised that inhibition of mitochondrial fission would be enough to block the normal functioning of mitochondrial metabolism. Indeed, it has been shown that a DRP1 mutant that inhibits mitochondrial fission increases glucose uptake and lactate production, and decreases ATP production [14]. Thus, we next aimed to measure the glycolytical function and the mitochondrial respiration in MCF7 cells exposed to mDIVI1. The extracellular acidification rate (ECAR) and the oxygen consumption rate (OCR) were measured using an XF96 Extracellular Flux Analyser (Figures 3A and 4A). Basal glycolysis, glycolytic capacity and glycolytic reserve were calculated after addition of glucose, oligomycin and 2-deoxyglucose (2DG) into the media. Surprisingly, exposure to mDIVI1 did not have a significant effect on basal glycolysis. However, the glycolytic capacity and glycolytic reserve of MCF7 cells was reduced after treatment with mDIVI1 (Figure 3B). That is, treatment with mDIVI1 for 48 hours blocked the increase of the ECAR usually linked to the oligomycin-induced inhibition of mitochondrial complex $\mathrm{V}$ of the electron transport chain, indicating that mDIVI1-treated MCF7 either have less ATP demand or have a less efficient mitochondrial oxidative phosphorylation than vehicletreated cells. Thus, to measure basal respiration, ATP production, maximal respiration and spare respiratory capacity, oxygen consumption was also calculated after addition of oligomycin, FCCP and antimycin/rotenone into glucose-containing media. In fact, exposure to mDIVI1 for 48 hours significantly reduced the oxygen consumption linked to basal respiration, ATP production and to a lesser extent, maximal respiration at higher concentrations (Figure 4B). However, it slightly increased the spare respiratory capacity of MCF7 cells after treatment with all mDIVI1 concentrations, suggesting that basal respiration in mDIVI1-treated is further from its theoretical maximum than vehicle-treated cells. The OCR versus ECAR graph was also plotted to have an indication of the metabolic state of the cell. mDIVI1 treatment also decreased dose-dependently the OCR/ECAR ratio of
MCF7 cells compared to vehicle, indicating that mDIVI1treated MCF7 cells are less aerobic and metabolically less active (Figure 4C). Thus, mDIVI1-induced inhibition of mitochondrial fission functionally targets oxidative phosphorylation and also the glycolytic function of MCF7 cells, transforming them into cells with lower mitochondrial energetic needs.

To identify differentially regulated proteins upon treatment with mDIVI1, MCF7 cells were exposed for 48 hours to either vehicle or $10 \mu \mathrm{M}$ mDIVI1 and cell lysates were subject to label-free quantitative proteomics. Following protein digestion with trypsin, peptide fractions were processed on an LTQ-Orbitrap XL mass spectrometer. Those peptides identified were further analysed to find proteomic changes between mDIVI1-treated and vehicletreated MCF7 cells, as described before [15]. To define differential regulation, those identified proteins that showed a fold change difference of 1.5 or higher, and $p$ values of $<0.05$ (ANOVA) compared to vehicle were considered. First, we searched the proteomics datasets for changes in proteins involved in metabolism (summarised in Table 1). The expression of several glycolytic enzymes, as well as pentose phosphate pathway enzymes and enzymes involved in mitochondrial metabolism were found to be down-regulated in mDIVI1-treated MCF7 cells compared to vehicle treatment. Of note, hexokinase, which generates glucose 6-phosphate from glucose for the glycolytic and the pentose phosphate pathways, was infinitely downregulated. In fact, the first two enzymes of the oxidative branch of the pentose phosphate pathway were also found downregulated in mDIVI1-treated cells compared to vehicle-treated cells (Table 1). That may possibly translate into a loss of antioxidant power, as the pentose phosphate pathway is a major source of NADPH. Furthermore, components of the complex III and V of the electron transport chain were found to be highly downregulated in mDIVI1-treated MCF7 cells (Table 1). To obtain additional functional insights into pathways that are differentially regulated in mDIVI1-treated MCF7 cells, bioinformatics analysis of the proteomics datasets were conducted. All proteins were analysed using the Ingenuity Pathway Analysis software (IPA) to seek altered canonical pathways and toxicity functions. Of note, amongst the altered pathways identified by IPA in the mDIVI1-treated cells were pathways involved in metabolism such as pentose phosphate pathway, mTOR signalling, AMPK and protein kinase A signalling, fatty acid biosynthesis, cholesterol and palmitate biosynthesis, mevalonate pathway and ketolysis. Likewise, the toxicity functions found to be altered in mDIVI1-treated MCF7 cells compared with vehicle treatment included decreases in permeability transition of mitochondria and mitochondrial membrane, fatty acid metabolism and cholesterol biosynthesis (Table 2). Thus, besides mitochondrial respiration and glycolysis, fatty acid metabolism also seems to be altered in mDIVI1- 
A

\section{Mitochondral mass}

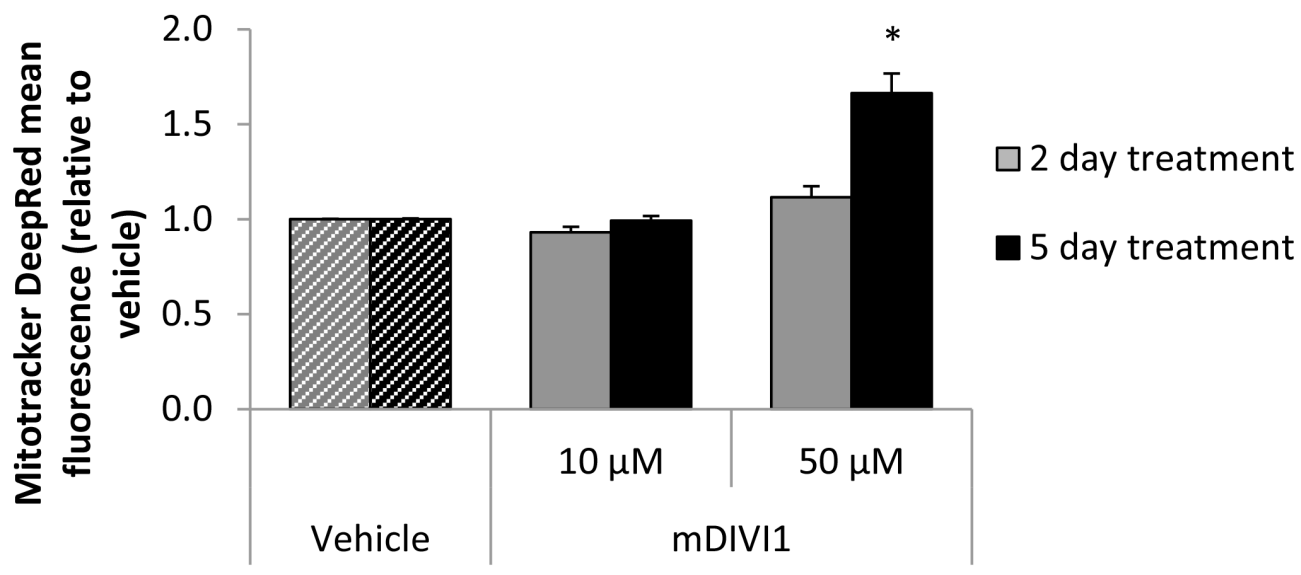

B

Mitochondrial superoxide

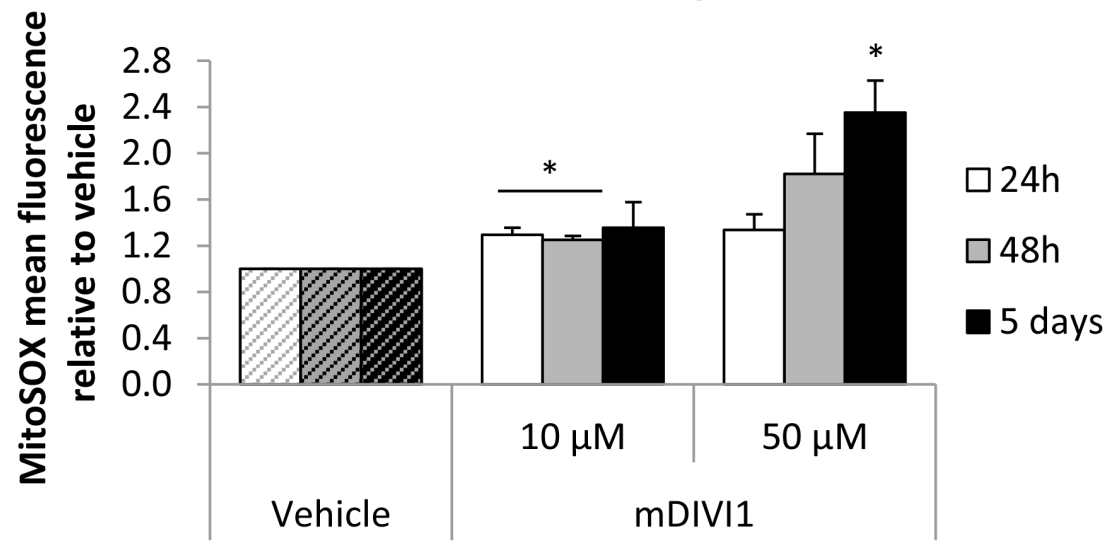

C

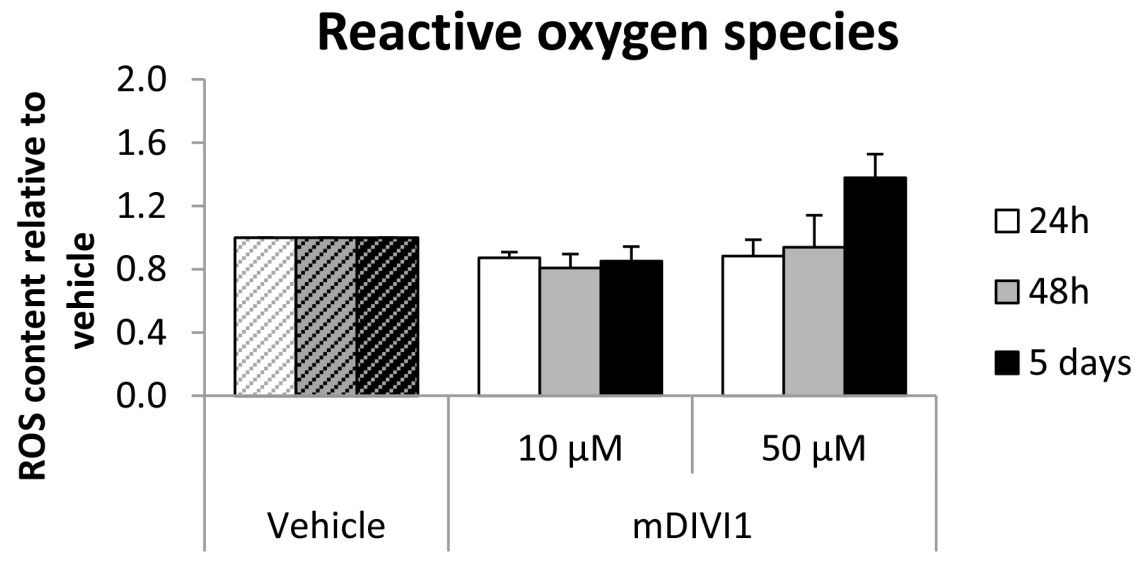

Figure 2: Treatment of MCF7 cells with mDIVI1 significantly increases their mitochondrial mass and mitochondrial oxidative stress at $50 \mu \mathrm{M}$. (A) Mitotracker Deep Red mean intensity significantly increased after 5 days of treatment with $50 \mu \mathrm{M}$ of mDIVI1. (B) Mitochondrial superoxide production significantly increased after 24 hour, 2 and 5 days of treatment. (C) Total ROS levels were not altered after exposure to mDIVI1. Data represented as MEAN \pm SEM. One sample $t$ test, ${ }^{*} p$ value $<0.05,{ }^{* *} p$ value $<0.01$ and ${ }^{* * * *} p$ value $<0.001 . N=3$. 
A

B
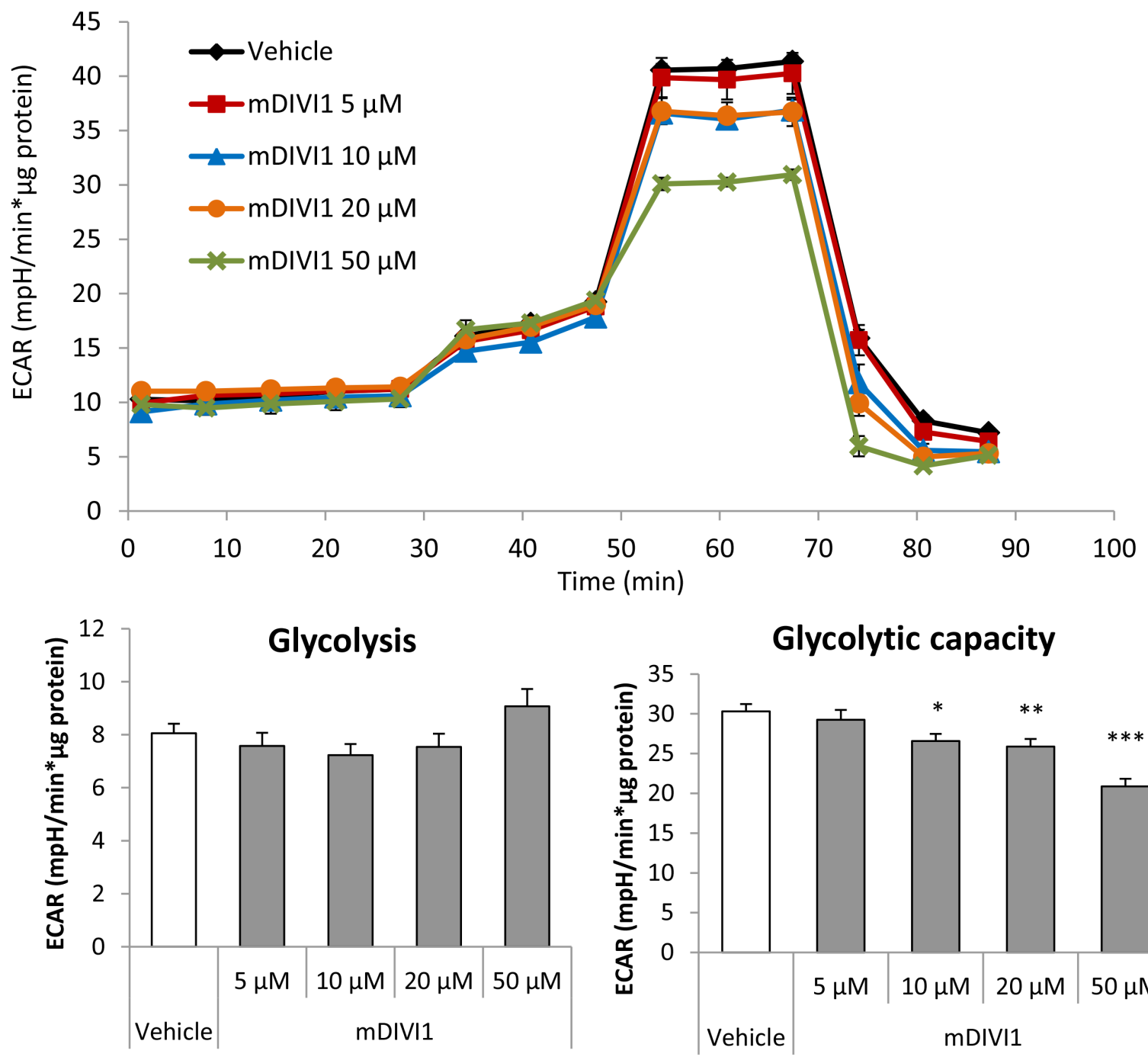

Glycolytic capacity
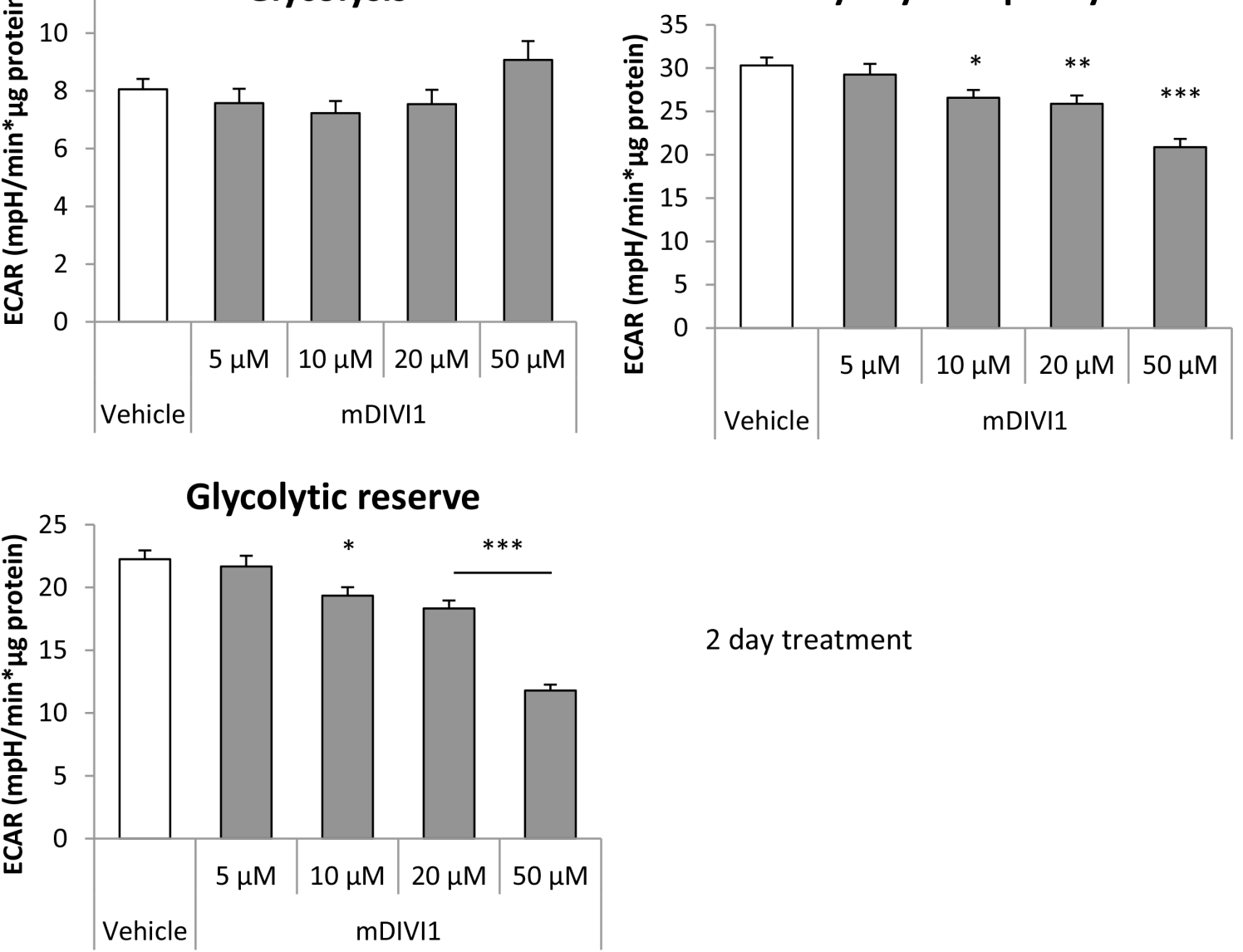

\section{2 day treatment}

Figure 3: mDIVI1 treatment significantly reduces the glycolytic capacity of MCF7 cells without having an effect on basal glycolysis, hence decreasing their glycolytic reserve. (A) Extracellular acidification rate (ECAR) of MCF7 cells treated with either vehicle or mDIVI1 for 48 hours and assessed using the Seahorse XFe96 Analyzer. (B) Treatment with $\geq 10 \mu \mathrm{M}$ mIVI1 for 48 hours blocks the increase of ECAR usually linked to oligomycin inhibition of the mitochondrial respiration, indicating a significant reduction in glycolytic capacity and reserve. mDIVI1-treated cells are using glycolysis closer to its maximum capacity compared to vehicle-treated cells. Data represented as MEAN \pm SEM. One way ANOVA, Dunnett's multiple comparisons test, ${ }^{*} p$ value $<0.05,{ }^{* *} p$ value $<0.01$ and ${ }^{* * *} p$ value $<0.001 . N \geq 3$. 

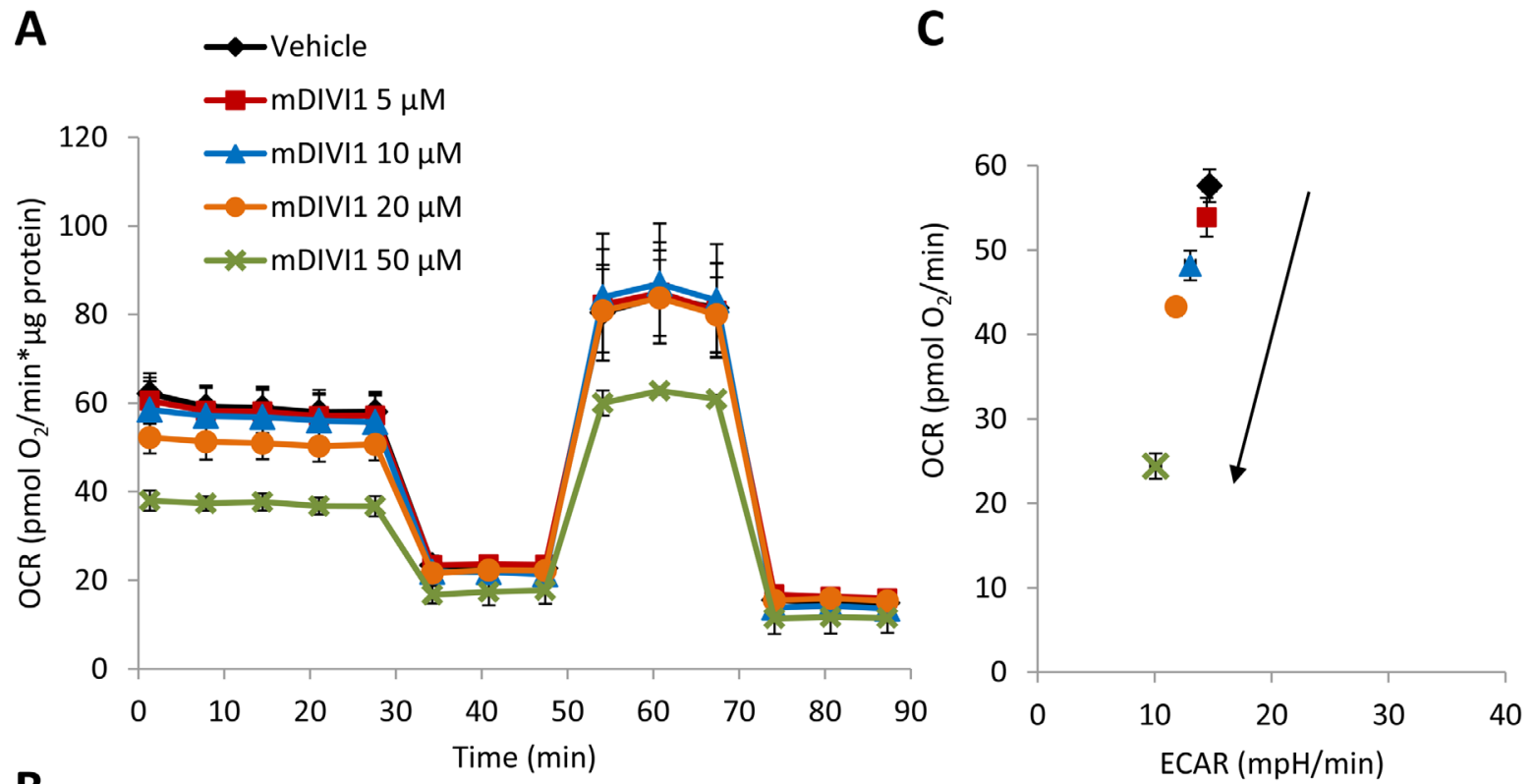

B
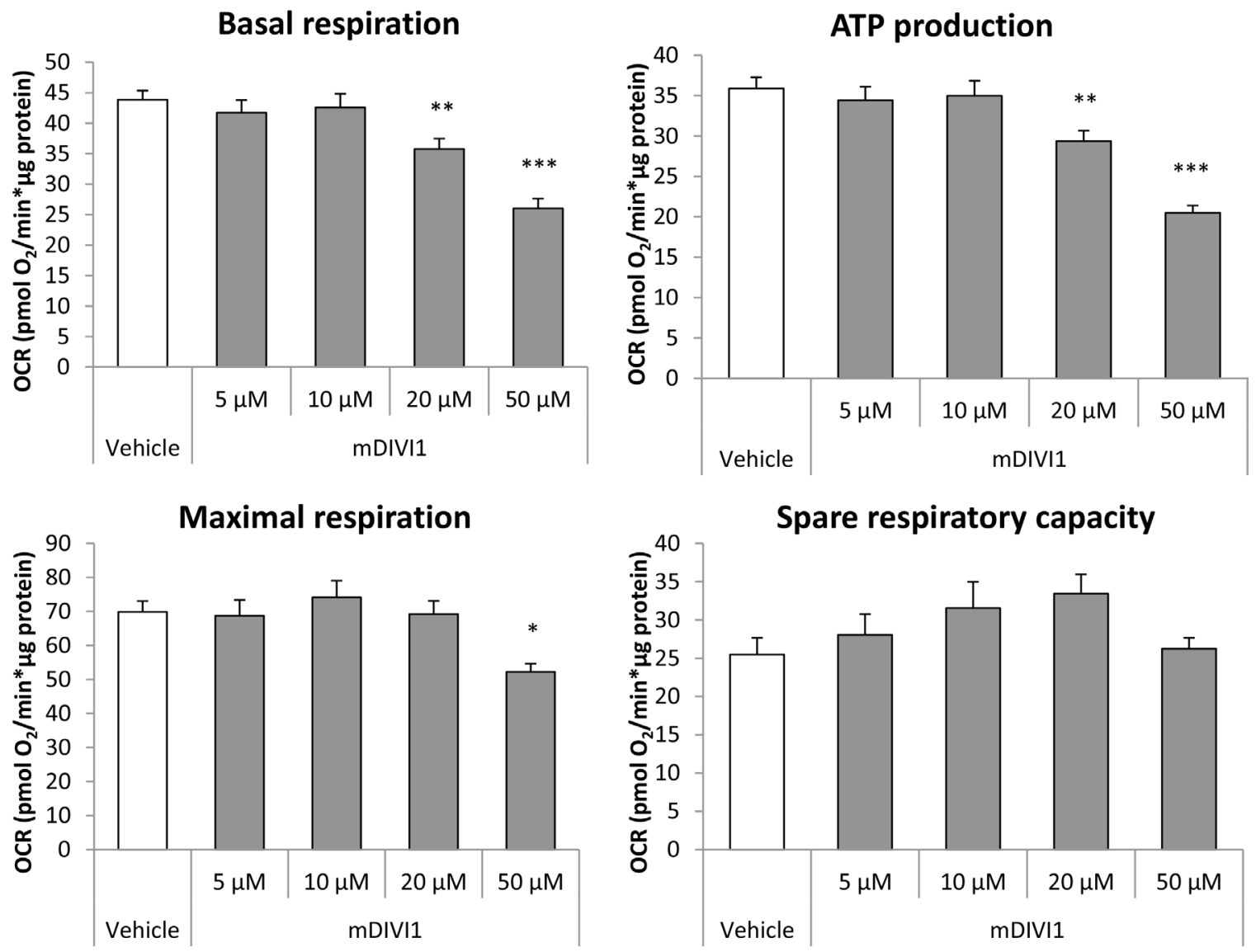

Figure 4: mDIVI1 treatment significantly reduces the respiration and ATP production of MCF7 cells but increases their spare respiratory capacity. (A) Oxygen consumption rate (OCR) of MCF7 cells treated with either vehicle or mDIVI1 for 48 hours and assessed using the Seahorse XFe96 Analyzer. (B) Treatment with mDIVI1 for 48 hours decreases the oxygen consumption linked to basal respiration, ATP production and to a lesser extent, maximal respiration. However it increases the spare respiratory capacity of MCF7cells, suggesting that mDIVI1-treated cells may be able to manage better an energetic crisis. (C) mDIVI1 treatment decreases the OCR/ECAR ratio of MCF7 cells indicating that mDIVI1-treated MCF7 cells are less aerobic and less metabolically active. Data represented as MEAN \pm SEM. One way ANOVA, Dunnett's multiple comparisons test, ${ }^{*} p$ value $<0.05,{ }^{* *} p$ value $<0.01$ and ${ }^{* * *} p$ value $<$ 0.001. $N=3$. 
Table 1: Changes in the expression of enzymes involved in several cellular metabolic pathways after exposure of MCF7 cells to mDIVI1 for $\mathbf{4 8}$ hours as measured by label-free quantitative proteomics

\begin{tabular}{|c|c|c|}
\hline Glycolysis & & $10 \mu \mathrm{M}$ mDIVI1 \\
\hline Hexokinase 1 & HK1 & $\downarrow$ Infinite \\
\hline Fructose-bisphosphate aldolase A & ALDOA & $\downarrow 2.88$ \\
\hline Enolase 1 & ENO1 & $\downarrow 6.63$ \\
\hline \multicolumn{3}{|l|}{ Post-Glycolysis Processes } \\
\hline Pyruvate dehydrogenase & PDHB & $\uparrow 1.63$ \\
\hline \multicolumn{3}{|l|}{ Pentose Phosphate Pathway } \\
\hline 6-phosphogluconolactonase & PGLS & $\downarrow 1.56$ \\
\hline Phosphogluconate dehydrogenase & PGD & $\downarrow 3.91$ \\
\hline \multicolumn{3}{|l|}{ Transketolase } \\
\hline TCA Cycle & TKT & $\uparrow 2.06$ \\
\hline Citrate synthase, mitochondrial & $\mathrm{CS}$ & $\downarrow 1.51$ \\
\hline \multirow{2}{*}{ Isocitrate dehydrogenase } & IDH2 & $\uparrow 1.84$ \\
\hline & IDH3G & $\downarrow 1.51$ \\
\hline \multicolumn{3}{|l|}{ Oxidative Phosphorylation } \\
\hline NADH dehydrogenase (complex I) MT-ND5 & $\begin{array}{c}\text { NDUFV1 } \\
\uparrow 1.84\end{array}$ & $\downarrow 1.63$ \\
\hline Coenzyme Q - cytochrome c reductase (complex III) & UQCRC1 & $\downarrow 2569.35$ \\
\hline Cytochrome c oxidase (complex IV) & COX6A1 & $\uparrow 1.70$ \\
\hline ATP synthase (complex V) & ATP5O & $\downarrow 13.56$ \\
\hline \multicolumn{3}{|l|}{ Fatty Acid Oxidation } \\
\hline Acetyl-Coenzyme A acyltransferase 1, peroxisomal & ACAA1 & $\uparrow 3.96$ \\
\hline Acetyl-Coenzyme A acyltransferase 2, mitocondrial & ACAA2 & $\downarrow 2.09$ \\
\hline Long-chain-aldehyde dehydrogenase & ALDH3A2 & $\uparrow 4.40$ \\
\hline Delta-1-pyrroline-5-carboxylate dehydrogenase, mitochondrial & ALDH4A1 & $\uparrow 2.40$ \\
\hline 4-trimethylaminobutyraldehyde dehydrogenase & ALDH9A1 & $\uparrow 1.72$ \\
\hline Acyl-CoA dehydrogenase family member 9 , mitochondrial & ACAD9 & $\downarrow 1.94$ \\
\hline Carnitine O-palmitoyltransferase 2, mitochondrial & CPT2 & $\uparrow 3.15$ \\
\hline \multicolumn{3}{|l|}{ Fatty Acid Synthesis } \\
\hline Fatty acid synthase & FASN & $\uparrow 1.97$ \\
\hline Beta-ketoacyl-ACP synthase & OXSM & $\downarrow 1.61$ \\
\hline \multicolumn{3}{|l|}{ Ketolysis/Ketogenesis } \\
\hline Acetyl-CoA acetyltransferase, mitochondrial & ACAT1 & $\downarrow 2.40$ \\
\hline 3-oxoacid CoA-transferase 1, mitochondrial & OXCT1 & $\uparrow 1.64$ \\
\hline 3-hydroxy-3-methylglutaryl-CoA synthase 1 & HMGCS1 & $\uparrow 4.09$ \\
\hline \multicolumn{3}{|l|}{ Lipid Metabolism (Other) } \\
\hline short/branched chain specific acyl-CoA dehydrogenase & ACADSB & $\downarrow 2.92$ \\
\hline Cytochrome P450 1B1 & CYP1B1 & $\uparrow 19.96$ \\
\hline Cytochrome P450, family 1, subfamily A, polypeptide 1 & CYP1A1 & $\uparrow 190.30$ \\
\hline Lanosterol $14 \alpha$-demethylase & CYP51A1 & $\uparrow 3.41$ \\
\hline Lanosterol synthase & LSS & $\downarrow 1.82$ \\
\hline Farnesyl-diphosphate farnesyltransferase 1 & FDFT1 & $\downarrow 1.50$ \\
\hline Isopentenyl-diphosphate delta isomerase & IDI1 & $\downarrow 1.79$ \\
\hline
\end{tabular}


Table 2: Pathway analysis of differentially expressed proteins in MCF7 cells treated with mDIVI1 compared to vehicletreated cells

\begin{tabular}{|c|c|c|c|}
\hline Ingenuity canonical pathways & $-\log (p$-value $)$ & Z score & Ratio \\
\hline Remodeling of Epithelial Adherens Junctions & 13.6 & -0.30 & 0.348 \\
\hline Actin Cytoskeleton Signaling & 11.5 & -1.15 & 0.176 \\
\hline Germ Cell-Sertoli Cell Junction Signaling & 9.79 & & 0.183 \\
\hline EIF2 Signaling & 9.4 & -0.83 & 0.173 \\
\hline Epithelial Adherens Junction Signaling & 8.9 & & 0.189 \\
\hline Tight Junction Signaling & 8.68 & & 0.175 \\
\hline Integrin Signaling & 8.46 & -0.36 & 0.156 \\
\hline ILK Signaling & 8.38 & 0.73 & 0.161 \\
\hline Regulation of eIF4 and p70S6K Signaling & 8.17 & -1.41 & 0.175 \\
\hline Paxillin Signaling & 7.85 & -0.69 & 0.2 \\
\hline Caveolar-mediated Endocytosis Signaling & 7.42 & & 0.239 \\
\hline Sertoli Cell-Sertoli Cell Junction Signaling & 7.07 & & 0.156 \\
\hline Regulation of Actin-based Motility by Rho & 6.77 & -0.73 & 0.207 \\
\hline Phagosome maturation & 6.61 & & 0.178 \\
\hline Clathrin-mediated Endocytosis Signaling & 6.48 & & 0.143 \\
\hline Regulation of Cellular Mechanics by Calpain Protease & 5.75 & -2.24 & 0.236 \\
\hline RhoA Signaling & 5.26 & -0.94 & 0.158 \\
\hline Mitotic Roles of Polo-Like Kinase & 5.05 & 0.82 & 0.206 \\
\hline RhoGDI Signaling & 4.95 & 0.85 & 0.134 \\
\hline Protein Ubiquitination Pathway & 4.71 & & 0.114 \\
\hline Role of BRCA1 in DNA Damage Response & 4.66 & & 0.179 \\
\hline Leukocyte Extravasation Signaling & 4.62 & -0.63 & 0.122 \\
\hline mTOR Signaling & 4.53 & 0.63 & 0.123 \\
\hline Actin Nucleation by ARP-WASP Complex & 4.15 & -1.51 & 0.196 \\
\hline FAK Signaling & 4.1 & & 0.153 \\
\hline DNA Double-Strand Break Repair by Non-Homologous End Joining & 3.42 & & 0.357 \\
\hline Signaling by Rho Family GTPases & 3.35 & -0.41 & 0.102 \\
\hline Estrogen Receptor Signaling & 3.29 & & 0.125 \\
\hline Superpathway of Cholesterol Biosynthesis & 2.79 & & 0.222 \\
\hline Cleavage and Polyadenylation of Pre-mRNA & 2.69 & & 0.333 \\
\hline AMPK Signaling & 2.66 & -0.63 & 0.101 \\
\hline Palmitate Biosynthesis I & 2.62 & & 1 \\
\hline Fatty Acid Biosynthesis Initiation II & 2.62 & & 1 \\
\hline Calcium Signaling & 2.39 & -0.81 & 0.1 \\
\hline Cdc42 Signaling & 2.37 & -0.83 & 0.109 \\
\hline Rac Signaling & 2.36 & -0.83 & 0.112 \\
\hline Telomere Extension by Telomerase & 2.3 & & 0.267 \\
\hline RAN Signaling & 2.19 & & 0.25 \\
\hline Assembly of RNA Polymerase I Complex & 2.11 & & 0.333 \\
\hline ERK/MAPK Signaling & 2.07 & -0.47 & 0.0909 \\
\hline Cell Cycle Control of Chromosomal Replication & 2.04 & & 0.185 \\
\hline PAK Signaling & 2.02 & -0.30 & 0.11 \\
\hline
\end{tabular}


Protein Kinase A Signaling

Apoptosis Signaling

Pentose Phosphate Pathway

Death Receptor Signaling

Unfolded protein response

DNA Methylation and Transcriptional Repression Signaling

Mevalonate Pathway I

Ephrin Receptor Signaling

Agranulocyte Adhesion and Diapedesis

GM-CSF Signaling

Axonal Guidance Signaling

Gap Junction Signaling

Ketolysis

CDK5 Signaling

\begin{tabular}{ccc}
2.01 & -1.61 & 0.0775 \\
1.97 & -1.26 & 0.114 \\
1.97 & & 0.3 \\
1.88 & -1.26 & 0.11 \\
1.85 & & 0.132 \\
1.83 & & 0.2 \\
1.74 & & 0.25 \\
1.67 & -1.39 & 0.0872 \\
1.61 & & 0.0857 \\
1.59 & -0.38 & 0.11 \\
1.56 & & 0.0703 \\
1.52 & & 0.0854 \\
1.37 & & 0.286 \\
1.3 & -1.34 & 0.0918 \\
\hline
\end{tabular}

\section{MDIVI1 inhibits migration of MCF7 cells}

It is though that CSCs are responsible for metastatic spread and growth. Emerging evidence highlights the role of mitochondrial dynamics in tumour cell dissemination [3]. These studies suggest that mitochondria are transferred to sites with high-energy demand. In migrating cancer cells mitochondria accumulate at the leading edge where processes requiring high energy occur, such as formation of focal adhesions. Fission seems to be a prerequisite for that efficient relocation of mitochondria. Indeed, upregulation/activation of DRP1 is associated with a migratory and invasive phenotype in cancer [3]. Thus, we next sought to assess whether mDIVI1-induced inhibition of DRP1 would decrease the migration of MCF7. To that purpose we performed a scratch assay, were cells were cultured in the presence of either vehicle or mDIVI1. The scratch was monitored over time and the percentage of wound closure was measured as described previously [16]. Exposure to 10 and $50 \mu \mathrm{M}$ mDIVI1 significantly reduced the percentage of wound closure compared to vehicletreated cells on a $14 \%$ and $44 \%$ respectively, therefore indicating a clear mDIVI1-induced dose-dependent reduction in MCF7 cell migration (Figure 6).

In addition, amongst the altered pathways identified by IPA in the mDIVI1-treated cells, there were pathways involved in cell motility such as actin cytoskeleton signalling, regulation of actin-based motility by rho, regulation of cellular mechanics by calpain protease (clearly predicted to be inactivated), cdc42 or RhoA signalling, and PAK signalling (Table 2). Likewise, some of the biological functions identified by IPA as affected by mDIVI1 involved cellular movement, migration and invasion, which in most cases was predicted to be decreased (Table 3). 


\section{MDIVI1 inhibits signalling pathways required for CSC survival in MCF7 cells}

Mitochondrial fusion and fission may also have an impact in signalling pathways that regulate stem cell proliferation and survival [17]. Thus, we next decided to determine how several signalling pathways that regulate stemness were affected by mDIVI1 exposure. For that purpose, a range of reporter MCF7-GFP cell lines were generated, including MCF7-GFP-GLI(luc), MCF7GFP-Rbp/Jk(luc), MCF7-GFP-SMAD(luc) and MCF7GFP-TCF/LEF(luc), to asses Hedgehog signalling, Notch signalling, TGF $\beta$ signalling and Wnt signalling respectively. These cell lines were exposed to either mDIVI1 or vehicle and assessed for luciferase activity. For the first time, we show that mDIVI1 treatment significantly inhibited all stem-related signalling pathways in a dose-dependent manner at most of the time points

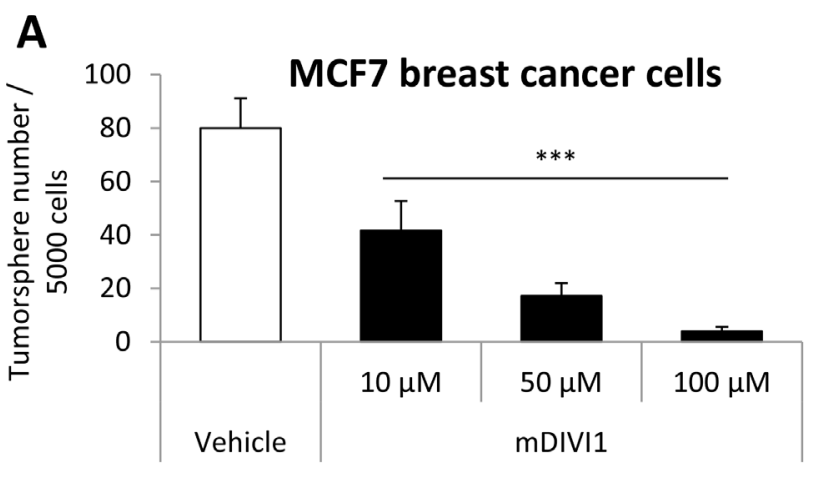

B

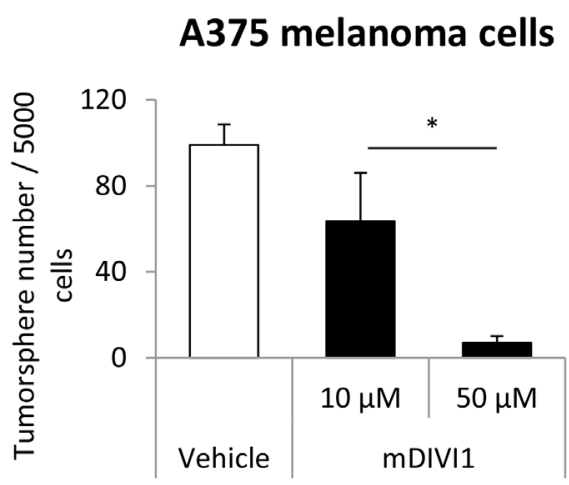

C
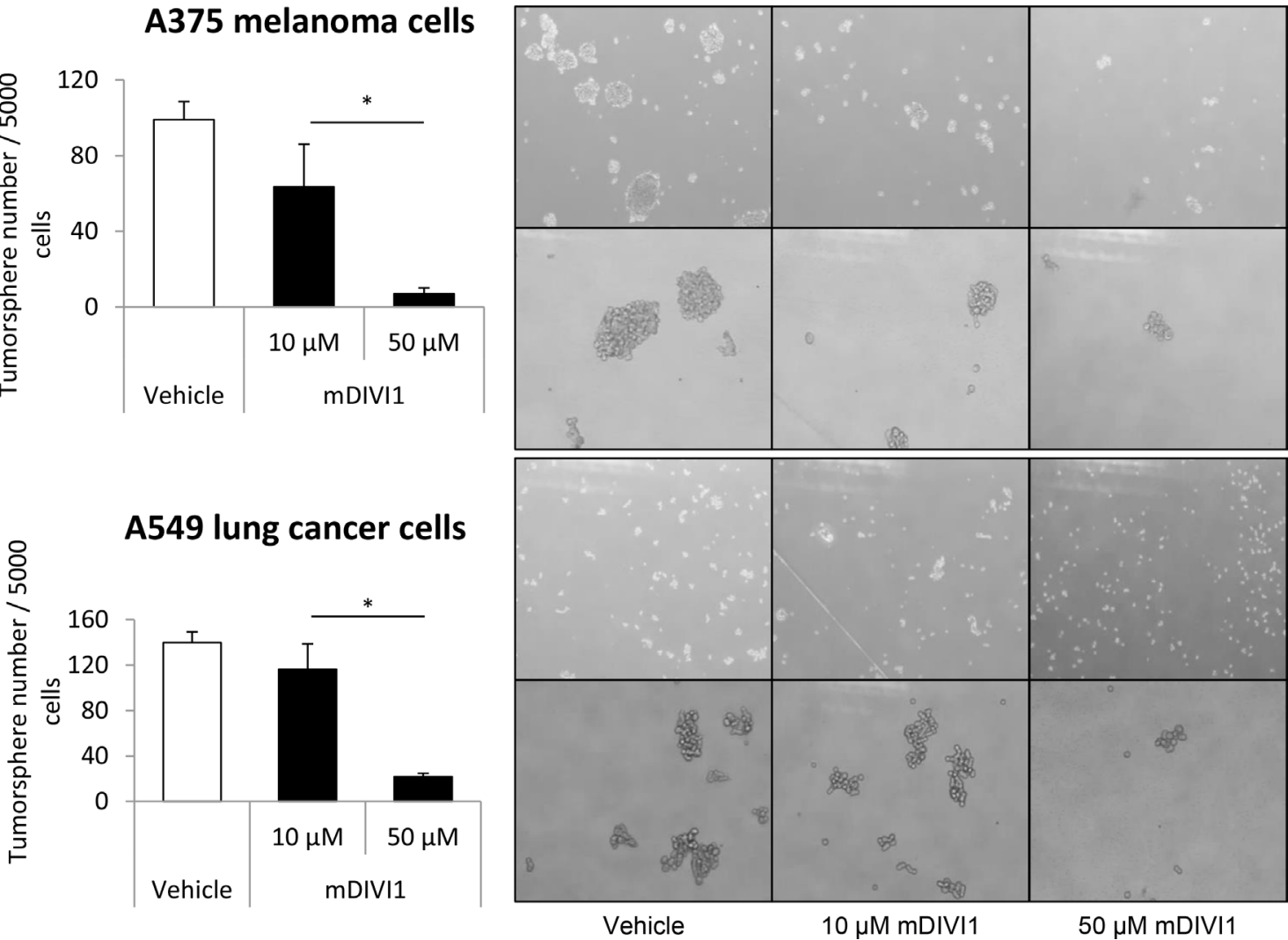

Figure 5: mDIVI1 treatment significantly reduces MCF7 tumorsphere formation, as well as the formation of $\mathrm{A375}$ melanoma and A549 lung tumorspheres. (A) Five days of treatment with the mitochondrial fission inhibitor mDIVI1 decreased tumorsphere number by $50 \%$ at a concentration of $10 \mu \mathrm{M}$ by $80 \%$ at $50 \mu \mathrm{M}$ and $95 \%$ at $100 \mu \mathrm{M}$. One-way ANOVA, post test for linear trend, ${ }^{* * *} p$ value $<0.001 . N=3$. (B) Five days of treatment with the mitochondrial fission inhibitor mDIVI1 dose-dependently decreased tumorsphere formation efficiency also in a melanoma (A375) and a lung cancer (A549) cell line. Data represented as MEAN \pm SEM. Oneway ANOVA, post test for linear trend ${ }^{*} p$ value $<0.05 N=2$. (C) Representative image of A375 melanoma and A549 lung tumorspheres after treatment with either vehicle or mDIVI1. Inhibition of DRP1 with mDIVI1 decreased tumorsphere number and also tumorsphere size. 
evaluated. Particularly, $50 \mu \mathrm{M}$ mDIVI1 strikingly caused the inhibition of the Hedgehog/GLI pathway, the Notch/ Rbp pathway, the Wnt/TCF pathway and the TGF $\beta /$ SMAD pathway 2 and 5 days after the start of the treatment (Figure 7).

Moreover, STAT3 (-1.83), EphA2 (-1.74) and BMP7 $(-47.17)$, all proteins involved in signalling pathways associated with cancer stem cell activities, were also found to be down-regulated in the proteomics datasets. Ephrin receptor signalling was specifically found to be significantly altered in mDIVI1-treated MCF7 cells compared to vehicle, with a negative $\mathrm{z}$ score indicating a slight inhibition of this pathway (Table 2). Likewise, TGF $\beta$ was identified by IPA as an upstream regulator predicted to be downregulated ( $z$ score $=-1.934$ ).

In summary, mDIVI1 inhibits all studied signalling pathways related with stemness in MCF7 cancer cells, confirming a suppression of the stem-like phenotype in these cells after treatment.

\section{MDIVI1 increases $\mathrm{CD}^{+} 4^{+} / \mathrm{CD}^{-} 4^{-}$population of MCF7 cells at high concentrations}

In human breast cancers, CSCs were first identified by the profile of expression of the cell surface marker CD $24^{-} / \mathrm{CD} 44^{+}[18]$. Thus, we next determined the expression levels of these CSC markers in MCF7 grown either as monolayers or under anchorage-independent conditions and exposed to mDIVI1 or vehicle. Surprisingly, the abundance of the CD24 $/ \mathrm{CD} 44^{+}$ subpopulation of MCF7 cells was not reduced after exposure to 10 or $50 \mu \mathrm{M}$ mDIVI1 compared to vehicle in cells grown as monolayers. Moreover, treatment of MCF7 cells in suspension with mDIVI1 unexpectedly increased the amount of CD24- $/ \mathrm{CD} 44^{+}$cells in a dosedependent fashion (Figure 8A and 8B). Thus, mDIVI1 exposure does not alter the proportion of CD24-/CD $44^{+}$ MCF7 CSCs grown as monolayers. However, under anchorage-independent growth conditions, the quantity of


B

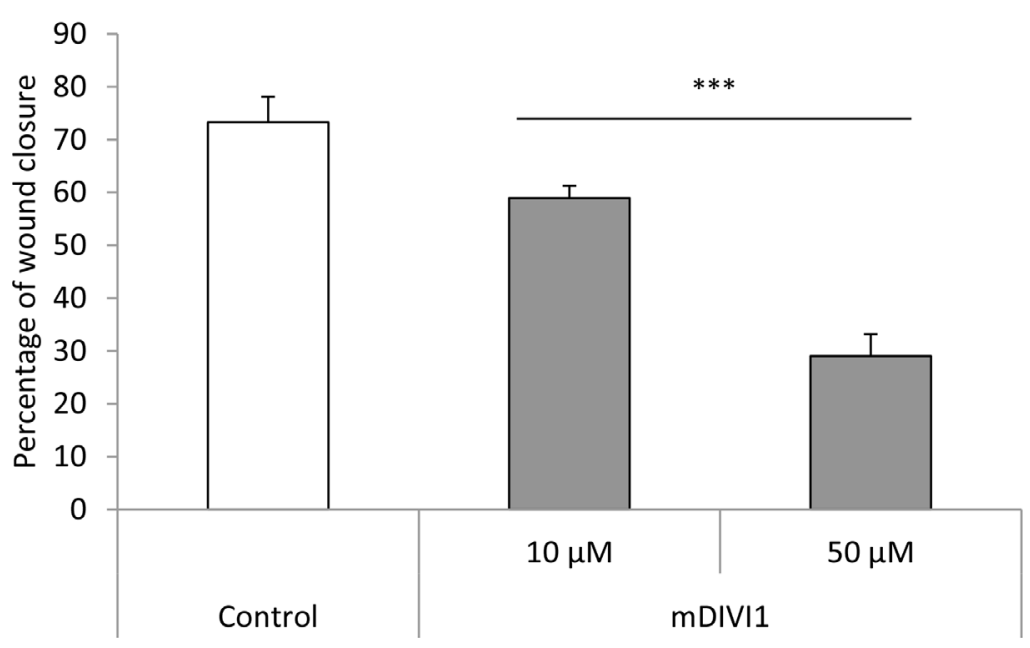

Figure 6: mDIVI1 treatment significantly reduces MCF7 migration in vitro. (A) Representative image of MCF7 cells after treatment with either vehicle or mDIVI1. Inhibition of DRP1 with mDIVI1 reduces scratch closure in vitro. (B) Two days of treatment with the mitochondrial fission inhibitor mDIVI1 significantly decreased the percentage of wound closure. Data represented as MEAN $\pm \mathrm{SEM}$. One-way ANOVA, post test for linear trend ${ }^{* * *} p$ value $<0.001 . N=4$. 
Table 3: Toxicity effects of differentially expressed proteins and biological functions affected by mDIVI1 treatment compared to vehicle-treated MCF7 cells

\begin{tabular}{|c|c|c|c|}
\hline Categories & Biological functions & $p$-Value & Activation z-score \\
\hline \multirow{6}{*}{ Cellular movement } & Invasion of cells & 0.00000127 & -1.806 \\
\hline & Invasion of tumour cell lines & 0.00000205 & -1.652 \\
\hline & Cell movement of tumour cell lines & 0.0000138 & -1.628 \\
\hline & Cell movement & 0.0000841 & -1.295 \\
\hline & Migration of tumour cell lines & 0.000153 & -1.164 \\
\hline & Cell movement of breast cancer cell lines & 0.000655 & -1.968 \\
\hline Cell Cycle, Cellular Movement & Cytokinesis & 0.0000132 & -0.669 \\
\hline \multirow{3}{*}{$\begin{array}{l}\text { Cell Cycle, DNA Replication, } \\
\text { Recombination, and Repair }\end{array}$} & Recombination of cells & 0.0000153 & -1.732 \\
\hline & Homologous recombination of cells & 0.0000327 & -1.732 \\
\hline & Homologous recombination & 0.000264 & -1.387 \\
\hline Cell Cycle & Cell cycle progression & 0.000218 & -1.762 \\
\hline $\begin{array}{l}\text { Cell Morphology, Cellular } \\
\text { Function and Maintenance, DNA }\end{array}$ & Repair of cells & 0.00145 & 1.921 \\
\hline $\begin{array}{l}\text { Replication, Recombination, and } \\
\text { Repair }\end{array}$ & Double-stranded DNA break repair of cells & 0.0035 & 2.069 \\
\hline \multirow{2}{*}{ Cell Morphology } & Shape change of tumour cell lines & 0.00354 & -1.446 \\
\hline & Cell spreading & 0.00383 & -1.633 \\
\hline \multirow{2}{*}{$\begin{array}{l}\text { Cellular Assembly and } \\
\text { Organization }\end{array}$} & Formation of cytoskeleton & 0.000722 & 1.95 \\
\hline & Formation of nucleus & 0.00185 & -2 \\
\hline $\begin{array}{l}\text { Cellular Function and } \\
\text { Maintenance }\end{array}$ & Endocytosis & 0.000264 & -1.832 \\
\hline $\begin{array}{l}\text { Cellular Assembly and } \\
\text { Organization }\end{array}$ & Formation of cytoskeleton & 0.000722 & 1.95 \\
\hline Gene Expression & Transcription & 0.000829 & 1.993 \\
\hline $\begin{array}{l}\text { DNA Replication, } \\
\text { Recombination, and Repair }\end{array}$ & Double-stranded DNA break repair & 0.000932 & 2.123 \\
\hline Cell Death and Survival & Cell viability & 0.00417 & -2.813 \\
\hline \multicolumn{2}{|l|}{ Ingenuity Toxicity Lists } & -log (p-value) & Ratio \\
\hline \multicolumn{2}{|l|}{ Cholesterol Biosynthesis } & 3.12 & 0.312 \\
\hline \multicolumn{2}{|c|}{ Fatty Acid Metabolism } & 3.02 & 0.133 \\
\hline \multicolumn{2}{|c|}{$\begin{array}{l}\text { Decreases Permeability Transition of Mitochondria and Mitochondrial } \\
\text { Membrane }\end{array}$} & 2.46 & 0.429 \\
\hline
\end{tabular}

CD24- ${ }^{-}{\text {CD } 44^{+}}^{-}$MCF7 cells increases in the mDIVI1 treatments in a dose-dependent manner.

It has been recently described that breast CSCs can exist in distinct states, a mesenchymal-like state and an epithelial-like state, being the first one characterised for its expression of $\mathrm{CD} 24^{-} / \mathrm{CD} 44^{+}$, with a mesenchymallike gene-expression profile and primarily quiescent [18]. Thus, we hypothesised that the increase in CD24-/CD $44^{+}$ MCF7 cells in suspension after treatment with mDIVI1 could respond to a change in these cells from an epithelial towards a more mesenchymal phenotype. We therefore searched for the expression of epithelial markers in MCF7 cells treated with either vehicle or mDIVI1. Immunoblot analysis of MCF7 cells treated with mDIVI1 revealed a reduction in the expression of E-cadherin after mDIVI1 treatment (Figure 8D). Decrease in the expression of other epithelial markers was observed also in the proteomics datasets, including EPCAM (-1.58), ZO-1 (-1.62), keratins 8 and $18(-5.24$ and -12.21$)$, CELSR2, involved in loss of polarization $(-1.6)$, F11 receptor $(-1.70)$, junction plakoglobin $(-1.65)$ and desmoplakin $(-4.58)$, although other epithelial markers such as collagen type IV was found to be upregulated by 1.9 fold. However, no mesencymal markers were identified as upregulated by proteomics. Notably, amongst the altered pathways identified by IPA in the mDIVI1-treated cells were 
pathways involved in cell to cell interaction and cell junctions such as remodeling of epithelial adherens junctions or epithelial adherens junctions signalling, tight junction signalling, gap junction signalling or FAK, integrin or paxillin signalling (Table 2). Thus, it seems mDIVI1 exposure induces the acquisition of a CD24-/CD44+ phenotype in MCF7 cells in suspension with loss of epithelial markers although no complete epithelial-to-mesenchymal transition is observed.

The DAPI staining that was performed in parallel to $\mathrm{CD} 24^{-} / \mathrm{CD} 44^{+}$staining to be used as quantification of viable cells, also revealed that there were no significant differences in cell viability between vehicle-treated cells and mDIVI1-treated cells under anchorage-independent conditions (Figure 8C). That is, mDIVI1-treated cells are not propagating as efficiently as vehicle-treated cells do, as observed in the tumorsphere formation assay, however they are more viable after mDIVI1 treatment in suspension than under attachment conditions. One
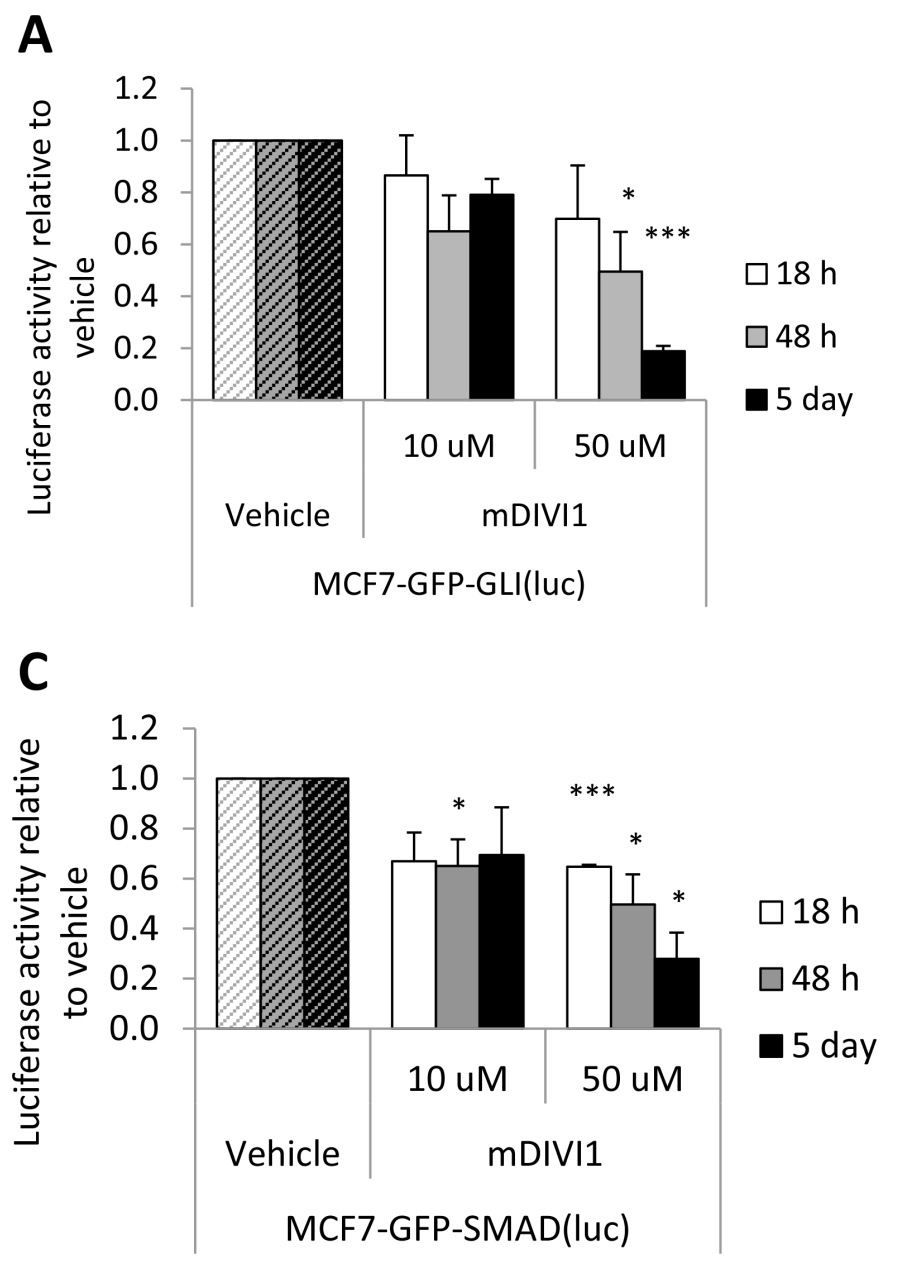

possible explanation to that observed behaviour is that they are becoming more quiescent. In fact, IPA analysis identifies cell cycle, $\mathrm{M}$ phase, cytokinesis, cell viability or cell proliferation as biological functions that are predicted to be decreased in mDIVI1-treated MCF7 cells compared to vehicle (Table 3). In addition, treatment with $50 \mu \mathrm{M}$ mDIVI1 showed frequent aberrations associated with failure of cytokinesis, which could be seen by DAPI immunofluorescence staining as an increase in binucleated cells (data not shown). Such phenomenon is typical of anti-mitotic drugs.

\section{DISCUSSION}

Recent findings show that mitochondrial morphology and function contribute to a stem-like phenotype, implying that mitochondrial fission and fusion are central players in CSC behaviour. The balance in mitochondrial dynamics is controlled by a small

B
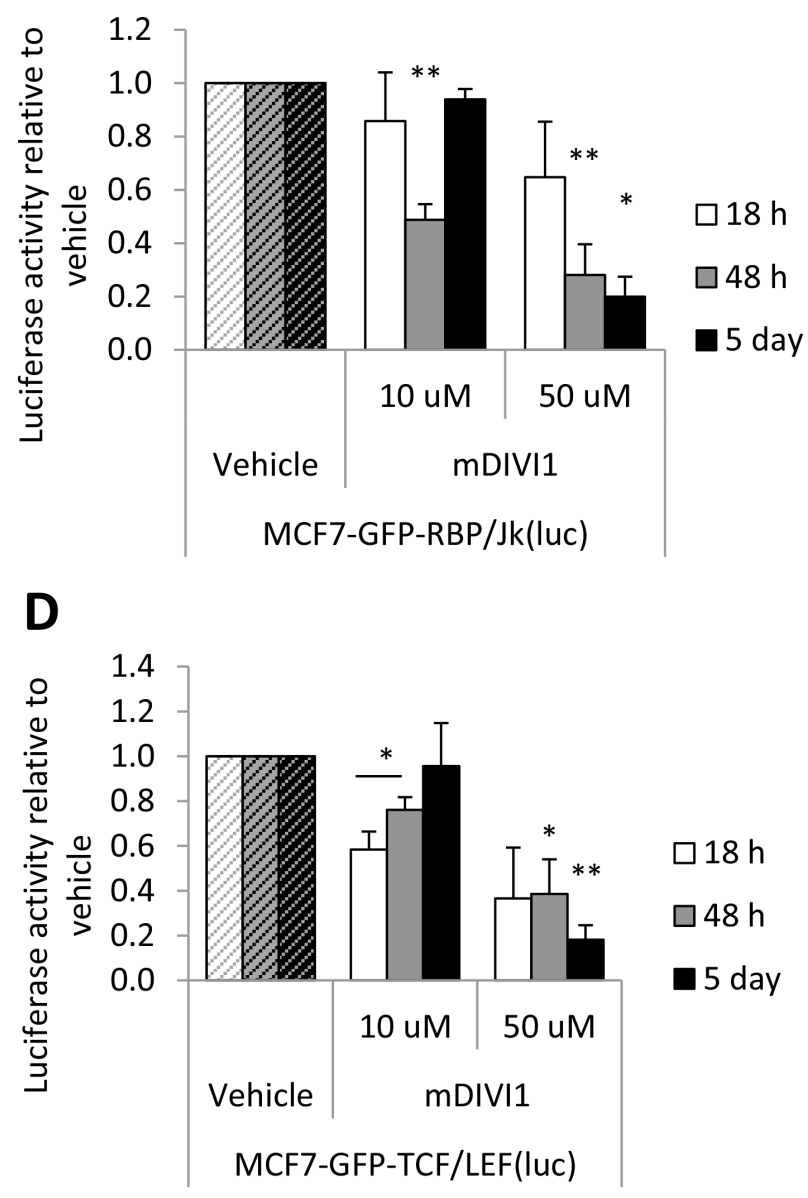

Figure 7: mDIVI1 treatment significantly inhibits stem-related signalling in MCF7 cells. (A) Hh/GLI (B) Notch/Rbp/Jk (C) TGF $/$ SMAD and (D) Wnt/TCF/LEF signaling were significantly inhibited after treatment with mDIVI1 at most time points assessed, particularly after exposure to $50 \mu \mathrm{M}$ mDIVI1. Data represented as MEAN \pm SEM. One sample $t$ test, ${ }^{*} p$ value $<0.05,{ }^{* *} p$ value $<0.01$ and ${ }^{* * *} p$ value $<0.001 . N=3$ 
cohort of regulators, such as DRP1. mDIVI1 is a small molecule inhibitor of DRP1 used in research for inhibiting mitochondrial fission (Figure 9). Emerging evidence demonstrates the implication of mitochondrial fission and in particular DRP1 in the tumorigenicity and maintenance of stem-like cells [7, 8]. However, a comprehensive study of the effects of mDIVI1 on CSC behaviour is still needed. Here, we tested the hypothesis that mDIVI1, through inhibition of mitochondrial fission, would induce loss of oxidative phosphorylation, therefore reducing the propagation of CSC, as they rely on their mitochondrial metabolism for their survival [11-13].

Our results show that mDIVI1-induced inhibition of DRP1 in MCF7 cells has a positive effect on their mitochondrial mass and production of mitochondrial reactive oxygen species, and that has a negative impact on mitochondrial metabolism. Mitochondrial fission plays a role in mitophagy, as it facilitates the elimination of defective organelles via isolation of selective parts of the mitochondria from the mitochondrial network. Therefore it is not surprising that after inhibiting DRP1, a slight increase in mitochondrial mass is observed. A few studies associate DRP1 and mitochondrial oxidative stress. Mitochondrial ROS levels are lower in DRP-overexpressing cells whereas those in a DRP1 mutant that inhibits mitochondrial fission are increased [14]. Moreover, when stress-induced hyperfusion of mitochondria happens in differentiated tissue, it increases mitochondrial ROS levels [19]. mDIVI1 also causes mitochondrial dysfunction and subsequent cell apoptosis in a DRP1-dependent manner in chemoresistant breast cancer cells [20]. In the current study, we show that mDIVI1-induced DRP1 inhibition alters the exctracellular acidification rate and oxygen consumption rate of MCF7 cells, transforming them in less aerobic, less metabolically active cells. mDIVI1 treatment seems to also have a big impact on lipid metabolism.

Previous studies performed in our lab strongly indicate that mitochondrial function has an implication in the propagation of CSCs [11-13]. We show here that mDIVI1-induced reduction in the respiration of MCF7 cells also impedes their propagation under anchorageindependent conditions. In addition, we show for the first time that mDIVI1 treatment decreases tumorsphere forming capacity in melanoma and lung cancer cell lines. However, the sphere assay has several weaknesses as even when plating them at a limiting dilution, individual spheres can be formed out of aggregation of cells rather than be generated through clonal expansion. Furthermore, more differentiated cells can also exhibit sphere-forming capability, and because tumorspheres themselves are heterogeneous, experiments using them should be interpreted as studies of mixed cell populations enriched for stem-like cells, not totally purified CSCs [21]. Taken together, these considerations made us use other approaches to asses CSC phenotype and activity, including the evaluation of several stemness-related pathways or the migratory ability of mDIVI1-treated MCF7 cells. Indeed, via generation of several reporter cell lines, we confirmed that mDIVI1 treatment was able to inhibit stemnessrelated signalling in MCF7, and mDIVI1 had also a negative effect on MCF7 cell motility.

Increased cell motility and invasion seem to require mitochondrial elongation and trafficking to the periphery of the cell. Several reports link mitochondrial localisation and cell motility by describing a redistribution of mitochondria toward the leading edge of cells during persistent migration. DRP1 silencing inhibits lamellipodia formation, a key step for cancer metastasis, by suppressing recruitment of mitochondria to those regions, and therefore decreasing the metastatic potential of breast cancer [22]. In order to be transported around the cells, mitochondria need to be transformed into small units, free from a tight network organization. Is during that process that DRP1 may play a role. Mitochondria might be needed at the leading edge as a source of energy, for calcium signalling, for the stabilisation of microtubules by ATP or even for the production of fatty acids and eicosanoids for membrane dynamics in the proximity of focal adhesions [23]. Our results show that treatment with mDIVI1 actually alters these processes, including oxidative phosphorylation and ATP production, lipid metabolism, and calcium and focal adhesion signalling (Table 2). Oxidative phosphorylation is actually required for the transfer of mitochondria to the cortical cytoskeleton. In respirationdeficient tumour cells, there is a lack of mitochondria in focal adhesion complexes, and invasion is impared [23]. Thus, via inhibition of mitochondrial fission and oxidative phosphorylation, mDIVI1 avoids distribution of mitochondria to the leading edge of the cell, and migration is impeded.

Indeed several studies point out that actin polymerization promotes mitochondrial fission. In addition, actin-depolymerizing drugs inhibit the recruitment of DRP1 to mitochondria and mitochondrial length is reduced [24]. Our proteomics results also show that one of the top canonical pathways affected by mDIVI1 is the actin cytoskeleton signalling.

Unexpectedly, we also found an increase in the abundance of $\mathrm{CD} 24^{-} / \mathrm{CD} 44^{+}$cells, which is a marker of CSC activity. Breast CSCs can exist in reversible heterogeneous states, a more epithelial-like state, and a more mesenchymal-like state, characterised for its expression of $\mathrm{CD} 24^{-} / \mathrm{CD}^{-} 4^{+}$, with marked reduction of oxygen consumption and increased quiescence [18]. However, although there was a clear reduction of epithelial markers after exposure to mDIVI1, as well as a reduction of mitochondrial respiration, we could not identify an over-expression of mesenchymal markers in mDIVI1treated MCF7 cells. Interestingly, during pluripotency reprogramming, epithelial-like cells display more 
A
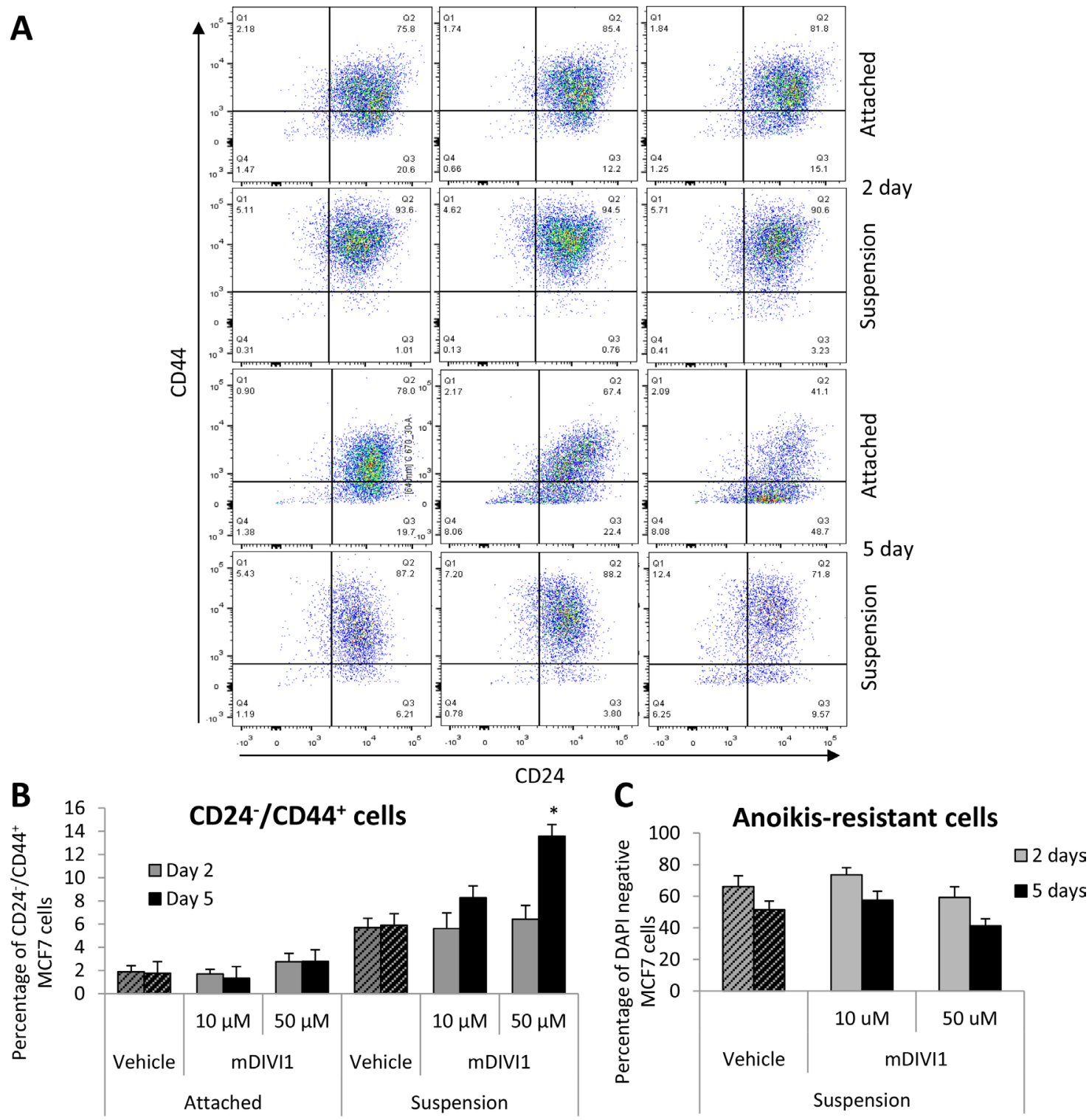

D

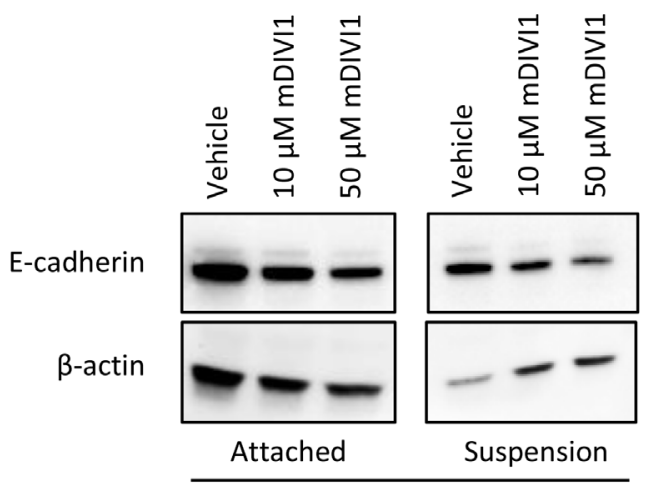

2 day treatment

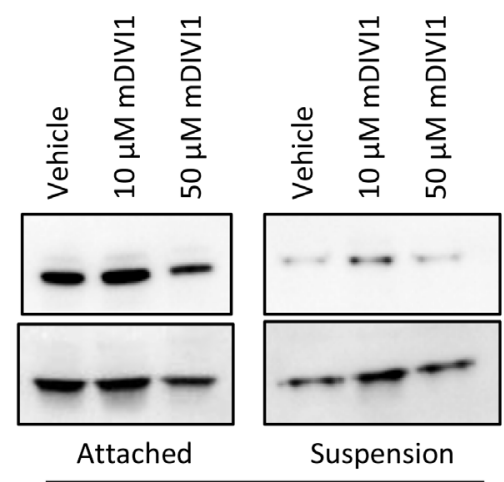

5 day treatment

Figure 8: $\mathrm{mDIVI1}$ treatment significantly increases the $\mathrm{CD}^{-} 4^{-} / \mathrm{CD}^{4} 4^{+}$population of $\mathrm{MCF} 7$ cells at high concentrations. (A) Representative graph showing CD24/CD44 staining of MCF7 cells treated with either vehicle or MDIVI1 and grown as either monolayers or in suspension for 2 or 5 days. (B) CD24 $/ \mathrm{CD} 44^{+}$subpopulation of MCF7 cells significantly increased after treatment with $50 \mu \mathrm{M}$ of mDIVI1 in suspension. (C) The percentage of anoikis-resistant cells did not significantly differ in mDIVI1 treatments from the vehicle treatment. One way ANOVA, Dunnett's multiple comparisons test, ${ }^{*} p$ value $<0.05,{ }^{* * *} p$ value $<0.01$ and ${ }^{* * *} p$ value $<0.001$. $N=4$ (D) Immunoblot analysis of MCF7 cells treated with mDIVI1 revealed a decrease of E-cadherin expression after mDIVI1 treatments. 
fragmented mitochondria, indicating that mitochondrial fission is critical for the acquisition of pluripotency [9].

Finally, compared to vehicle-treated cells, mDIVI1-treated cells seem to survive more in suspension than under attachment conditions, which could be related to the acquisition of a quiescent state. An exhaustive analysis of the cell cycle would be strongly advised to be able to tell whether these cells are actually acquiring a quiescent phenotype or are undergoing senescence. Our proteomics datasets did not identify many markers of senescence. Only LAMP1 was found to be upregulated in mDIVI1-treated cells compared to vehicle (2.83), although it is also involved in the processes of autophagy and mitophagy.

In summary, mDIVI1 has a negative impact on the anchorage-independent growth of MCF7 cells, on their migratory capacity and their signalling pathways related to stemness. However, it seems that mDIVI1 selects for a phenotype with loss of epithelial markers and features, such as cell to cell contact establishment, and in suspension enriches the CD24-/CD $44^{+}$population of cells. Several options could explain that behaviour. The first one could be that $\mathrm{CD} 24^{-} / \mathrm{CD} 44^{+}$expression is not representative of CSC activity in this scenario of mitochondrial fission inhibition, as for instance DRP1 effects on CSC behaviour are downstream of CD44, or mDIVI1 has a direct effect in CD24 loss or CD44 acquisition that does not have an impact on CSC survival. Another explanation would be that MCF7 cells enrich their CD24 ${ }^{-} / \mathrm{CD} 44^{+}$expression profile to counteract mDIVI1 effects, without being able to reverse the inhibition of the CSCs phenotype (tumorsphere formation efficiency, migration and stemness signalling) and therefore depleting CSCs.

Metabolism is materialising as a promising area for cancer treatment [25]. Nevertheless, the notion of targeting cancer mitochondrial metabolism or organelledriven adaptation is still underdeveloped. This study has been done in an effort to contribute to that concept, as a better understanding of processes engaged in the regulation of mitochondrial dynamics and their significance for CSC maintenance and propagation will provide us with the instruments to eventually alter them, offering new possible therapeutic approaches. It appears that CSCs have adopted metabolic control mechanisms to increase their survival and proliferation. In that scenario, mitochondrially-targeted drugs may represent promising new agents to interfere with tumour adaptation, ultimately eliminating CSCs [26]. The fact that mDIVI1 acts as a cytoprotective agent in non-tumour cells and that it would allow a reversible manipulation of mitochondrial morphology highlights its importance as a putative anticancer agent.

\section{Mitochondrial Cellular Dynamics}

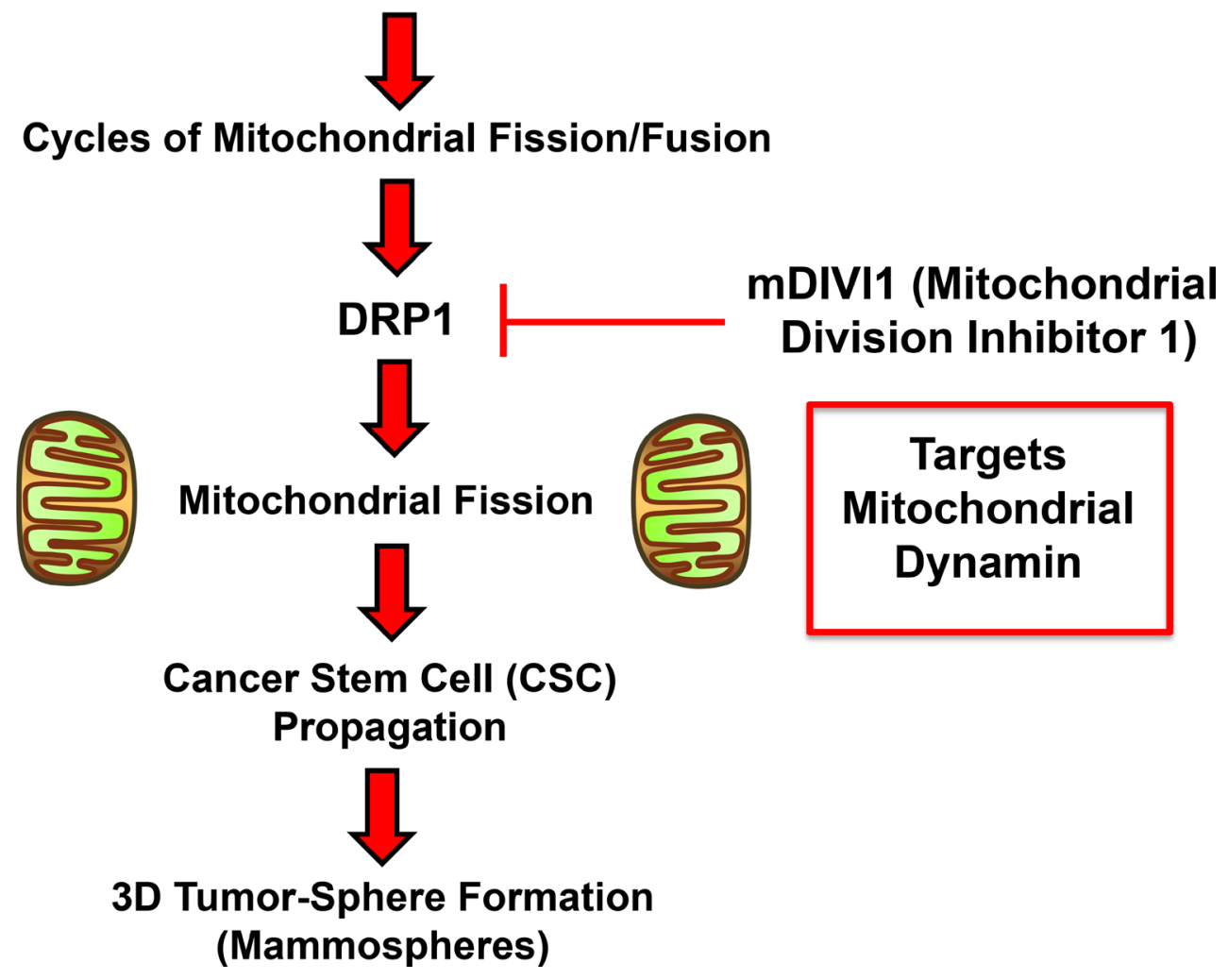

Figure 9: Schematic diagram summarizing our approach using mDIVI1 to investigate the role of mitochondrial fission/ fusion in CSC propagation. Note that mDIVI1 inhibits DRP1, a mitochondrial-specific dynamin protein. See text for further details. 


\section{MATERIALS AND METHODS}

\section{Cell culture}

Human MCF7 breast cancer cells were purchased from ATCC and maintained in complete media: DMEM (D6546, Sigma) supplemented with $10 \%$ fetal bovine serum (F7524, Sigma), 100 units $/ \mathrm{ml}$ of penicillin, 100 $\mu \mathrm{g} / \mathrm{ml}$, streptomycin (P0781, Sigma) and 1\% Glutamax (\#35050087, Life Technologies). For all experiments, cells were incubated in a $5 \% \mathrm{CO}_{2}$ atmosphere at $37^{\circ} \mathrm{C}$.

\section{Chemicals}

mDIVI1 or 3-(2,4-Dichloro-5-methoxyphenyl) 2,3-dihydro 2-thioxo-4(1H) quinazolinone (sc-215291, Santa Cruz), an inhibitor of mitochondrial division DRP1 and dynamin I, was used in this study at the concentrations indicated. Vehicle-treated cells (DMSO $0.5 \%$ ) were analysed in all experiments.

\section{Prestoblue viability assay}

Cell viability was measured using the resazurinbased PrestoBlue reagent (A-13261, ThermoFisher Scientific). Briefly, $5 \times 10^{3}$ MCF7 cells were seeded into 96-well black plates. When cells were attached mDIVI1 or vehicle were added to the cells. After 2 or 5 days, PrestoBlue reagent was added to the cells and incubated for 2 hours. Plates were finally read using a FluoStar Omega plate reader (BMG Labtech) at an excitation wavelength of 544 and an emission wavelength of 590 $\mathrm{nm}$. Background measurements were subtracted from all values.

\section{Mitochondrial mass quantification}

To measure mitochondrial mass, cells were stained with MitoTracker Deep Red (M22426, Invitrogen). Briefly, $2 \times 10^{5}$ MCF7 cells per well were seeded in 6-well plates. When cells were attached, mDIVI1 and vehicle treatments were added for 2 days or 5 days in triplicate. Cells were then incubated for $15 \mathrm{~min}$ at $37^{\circ} \mathrm{C}$ with the $10 \mathrm{nM}$ Mitotracker Deep Red probe diluted in PBS (D8662, Sigma). All subsequent steps were performed in the dark. Cells were washed in PBS, harvested, and resuspended in PBS. Mitotracker signal was quantified as mean fluorescent intensity of the viable cell population in a Fortessa flow cytometer (BD Bioscience). Results were analyzed using FlowJo software.

\section{Mitochondrial staining}

One hundred thousand MCF7 cells were seeded onto coverslips in 12-well plates. When cells were attached, mDIVI1 and vehicle treatments were performed in triplicate. After 2 days and 5 days, mitochondria were labeled by incubating cells for $15 \mathrm{~min}$ at $37^{\circ} \mathrm{C}$ with $25 \mathrm{nM}$ of MitoTracker DeepRed (M22426, Invitrogen) diluted in PBS (D8662, Sigma). Then, cells were washed with PBS, fixed in 2\% paraformaldehyde (28908, Thermo Scientific) in PBS for 30 minutes at room temperature and mounted with ProLong ${ }^{\circledR}$ Gold Antifade Mountant reagent with DAPI (P36935, Invitrogen, Inc.). Immunofluorescence pictures were taken in a Leica gated Stimulated Emission Depletion Microscopy (gSTED) with additional confocal and multi-photon illumination (room rg106).

\section{Levels of reactive oxygen species and mitochondrial superoxide}

For both assays, $2 \times 10^{5} \mathrm{MCF} 7$ cells were seeded in $35 \mathrm{~mm}$ plates. When cells were attached, either vehicle or mDIVI1 were added to the cells for 24,48 hours or 5 days in triplicate. Reactive oxygen species (ROS) production was measured using $\mathrm{CM}-\mathrm{H}_{2}$ DCFDA (C6827, Invitrogen). Briefly, cells were incubated for 20 minutes at $37^{\circ} \mathrm{C}$ with $1 \mu \mathrm{M} \mathrm{CM}-\mathrm{H}_{2} \mathrm{DCFDA}$ diluted in PBS and then placed in complete media for 20 minutes at $37^{\circ} \mathrm{C}$ in the dark, to render the dye fluorescent, according to the manufacturer. Mitochondrial superoxide was measured using MitoSOX Red Mitochondrial Superoxide Indicator (M36008, ThermoFisher Scientific). Briefly, cells were incubated for 10 minutes at $37^{\circ} \mathrm{C}$ in the dark with $5 \mu \mathrm{M}$ MitoSOX diluted in PBS, according to the manufacturer. ROS signal and MitoSOX signal was quantified as mean fluorescent intensity of the viable cell population in a Fortessa flow cytometer (BD Bioscience). Results were analysed using FlowJo software.

\section{Extracellular flux analysis and bradford assay}

Extracellular acidification rate (ECAR) and oxygen consumption rate (OCR) were measured in a XF96 Extracellular Flux Analyzer (Seahorse Biosciences). Briefly, $1 \times 10^{4}$ MCF7 cells per well were seeded in XF96 plates and incubated with complete medium. When cells were attached, mDIVI1 and vehicle treatments were added. Six replicates were run for each condition. After 48 hours, un-buffered DMEM XF medium supplemented with $2 \mathrm{mM}$ glutamine ( $\mathrm{pH}$ 7.4) was added to the cells, and placed in a $37^{\circ} \mathrm{C} \mathrm{CO}_{2}$-free incubator for 1 hour. Ten $\mathrm{mM}$ glucose, $1 \mu \mathrm{M}$ oligomycin and $100 \mathrm{mM}$ 2-deoxyglucose (2-DG) were injected into the media at different time points and ECAR was measured. Likewise, ECAR and OCR were quantified using unbuffered DMEM XF medium supplemented with $2 \mathrm{mM}$ glutamine, $2 \mathrm{mM}$ sodium pyruvate and $10 \mathrm{mM}$ glucose. One $\mu \mathrm{M}$ oligomycin, $0.9 \mu \mathrm{M}$ FCCP and $1 \mu \mathrm{M}$ rotenone and antimycin A were injected into the media at different time points and OCR and ECAR were measured. 
Cells were finally lysed with $0.1 \mathrm{M} \mathrm{NaOH}$ and protein lysate was subsequently stained with Quick Start ${ }^{\mathrm{TM}}$ Bradford 1× Dye Reagent (500-0205, BioRad) to normalise results. All parameters were calculated according to manufacturer.

\section{Tumorsphere formation assay}

A single cell suspension was prepared using enzymatic (1× Trypsin-EDTA, T3924, Sigma Aldrich), and manual disaggregation (25 gauge needle) [27]. Cells were plated at a density of 5000 cells/well in tumorsphere media (DMEM-F12/B27/EGF (20ng/ml)/Pen-Strep) in non-adherent conditions, in 6-well plates coated with (2-hydroxyethylmethacrylate) (poly-HEMA, P3932, Sigma) in the presence of mDIVI1 or vehicle. After 5 days of culture in a humidified incubator at $37^{\circ} \mathrm{C}$, tumorspheres bigger than $50 \mu \mathrm{m}$ were counted using an eye piece graticule.

\section{Scratch assay}

A hundred thousand MCF7 cells per well were seeded in 12-well plates. When cells were attached, a horizontal scratch was performed using the tip of a pipette. Immediately after, the media was removed and either fresh vehicle-containing media or mDIVI1-containing media were added to the cells for 48 hours. Four replicates were used for each condition. Cells were incubated in a $5 \%$ $\mathrm{CO}_{2}$ atmosphere at $37^{\circ} \mathrm{C}$ in an Incucyte ZOOM System (Essen Bioscience). The wound closure was monitored by taking pictures of each well every 4 hours. At the end of the treatment, pictures of the wells at 0 and 48 hours were analysed using Image J software to calculate wound closure according to the following formula:

Percentage wound closure $=\left[\left(\right.\right.$ wound $_{\text {area }}{ }_{0 \mathrm{~h}}-$ wound

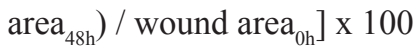

\section{Luciferase assay}

The Cignal Lenti reporter assay (luc) was used to monitor the activity of several signalling pathways in MCF7-GFP cells as explained previously [28]. Luciferase Assay System (E1501, Promega) was performed according to manufacturer's instructions. $1 \times 10^{4}$ MCF7 were seeded in black-walled 96 well plates. When cells were attached, drug treatments were added for 18, 48 hours and 5 days. After treatment, Luciferase Assay was performed according to manufacturer's instructions and light signal was acquired in the Xenogen VivoVision IVIS Lumina. Results were normalized by SRB staining of cells grown in 96-well plates in parallel.

\section{Sulforhodamine b (SRB) assay}

SRB (S9012, Sigma) measures total biomass by staining cellular proteins. After treatment, cells were fixed in 10\% tricloroacetic acid (T9159, Sigma) for 1 hour at $4^{\circ}$ C, stained with SRB (S9012, Sigma) for 15 minutes, and washed 3 times with $1 \%$ acetic acid (27225, Sigma). The incorporated die was solubilized with 10 mM Tris Base, pH 8.8 (T1503, Sigma). Absorbance was spectrophotometrically measured at $562 \mathrm{~nm}$ in a FluoStar Omega plate reader (BMG Labtech). Background measurements were subtracted from all values.

\section{CD24/CD44 expression}

Four hundred thousand MCF7 cells were seeded either in either a regular $10 \mathrm{~cm}$ dish or in five $15 \mathrm{~cm}$ dishes coated with (2-hydroxyethylmethacrylate) (poly-HEMA, P3932, Sigma) in the presence of mDIVI1 or vehicle for 2 days or 5 days. Following mDIVI1 treatment, MCF7 cells grown either in suspension or as monolayers were analysed for their expression of CD24 and CD44. The surviving fraction after 2 days and 5 days of growth was analyzed by FACS. MCF7 cells were either trypsinised or spun down and trypsinised and incubated with CD24 (IOTest CD24-PE, Beckman Coulter) and CD44 (APC mouse anti-human CD44, 559942, BD Pharmingen) for 15 minutes on ice. Cells were then rinsed twice in PBS, spun down, and resuspended with DAPI dye (D1306, Molecular probes) at $3 \mu \mathrm{M}$ in PBS for 10 minutes. Samples were then analyzed by FACS (Fortessa, BD Bioscence). Only the live cell population, identified using the DAPI staining, was analyzed for CD24/CD44 expression. Data were analyzed using FlowJo software. Only those cells expressing CD44 that did not express $\mathrm{CD} 24$ were considered to be the CD24 $/$ CD $44^{+}$CSC subpopulation.

\section{Western blotting}

Two million MCF7 cells were seeded either in regular $15 \mathrm{~cm}$ dishes or in five $15 \mathrm{~cm}$ dishes coated with (2-hydroxyethylmethacrylate) (poly-HEMA, P3932, Sigma) in the presence of mDIVI1 or vehicle for 2 days or 5 days. At the end of the treatment, cells in suspension were spun down and lysed and attached cells were directly lysed in RIPA lysis buffer (R0278, Sigma) containing proteinase inhibitors (05 892970 001 , Roche) and kept at $4^{\circ} \mathrm{C}$ for 20 minutes with rotation. Lysates were cleared by centrifugation for 10 minutes at 10,000 xg and supernatants were collected. Equal amounts of protein lysate, as determined by using the BCA protein assay kit (23225, Pierce) were diluted in SDS sample buffer and dry-boiled for 5 minutes before being separated by SDS-PAGE using 4-20\% gels (456-1094, Biorad). Samples were then blotted onto nitrocellulose membranes (170-4159, 
Biorad), blocked in 5\% milk in TBS-Tween 20 (P9416, Sigma) for 1 hour and probed with antibodies against E-cadherin (ab8995, Abcam) or $\beta$-actin (a2228, Sigma). Bound antibodies were detected using a horseradish peroxidase-conjugated secondary antibody (ab6789 and ab6721, Abcam) and signal was obtained using Supersignal West Pico chemiluminiscent substrate (34087, ThermoScientific). Pictures were taken in a ChemiDoc XRS with Image Lab Software (BioRad).

\section{Label-free semi-quantitative proteomics}

\section{Chemicals and sample preparation.}

Formic acid, trifluoroacetic acid, ammonium formate $(10 \mathrm{M})$, ammonium bicarbonate TCEP (Tris (2-carboxyethyl)phosphine hydrochloride), MMTS (Methyl methanethiosulfonate) and trypsin were all obtained from Sigma. HPLC gradient grade acetonitrile was obtained from Fisher Scientific. Briefly, $2 \times 10^{6}$ MCF7 cells were seeded in $15 \mathrm{~cm}$ plates until cells were attached. Cells were then treated with $10 \mu \mathrm{M}$ mDIVI1. As control, vehicle-treated cells were processed in parallel. After 48 hours of treatment, cells were lysed in RIPA buffer (R0278, Sigma) and kept at $4^{\circ} \mathrm{C}$ for 20 minutes with rotation. Lysates were cleared by centrifugation for 10 minutes at $10,000 \times \mathrm{g}$ and supernatants were collected and kept frozen at $-80^{\circ} \mathrm{C}$.

\section{Protein digestion}

Lysate samples were thawed to room temperature and their concentrations equalised to $1 \mu \mathrm{g} / \mu \mathrm{L}(50 \mu \mathrm{L}$ volume) with RIPA buffer, and further processed for trypsin digestion by sequential reduction of disulphide bonds with TCEP and alkylation with MMTS. Briefly, $1 \mu \mathrm{L}$ benzonase (Novagen) was added to the $50 \mu \mathrm{L}$ aliquot and placed on ice for 15 minutes. The sample was then taken to dryness using a SpeedVac, and resuspended in $22.5 \mu \mathrm{L}$ trypsin reaction buffer (40 $\mathrm{mM}$ ammonium bicarbonate and $9 \%$ acetonitrile). One $\mu \mathrm{L}$ of $50 \mathrm{mM}$ TCEP solution was added to each sample, mixed briefly and placed on a heater block at $60^{\circ} \mathrm{C}$ for 60 minutes. After cooling to room temperature, $0.5 \mu \mathrm{L}$ of $200 \mathrm{mM}$ MMTS solution was added to each sample and allowed to react for 15 minutes. Trypsin was added in two waves to ensure efficient digestion of the sample. Firstly, $20 \mu \mathrm{g}$ of sequencing grade trypsin was resuspended in $1800 \mu \mathrm{L}$ of trypsin reaction buffer; $225 \mu \mathrm{L}$ of this solution were added to each sample for digestion, and the reactions were left at $37^{\circ} \mathrm{C}$ overnight with shaking (600 rpm). The following morning, a further aliquot of trypsin was added. Two $\mathrm{ml}$ of trypsin reaction buffer was added to $20 \mu \mathrm{L}$ of sequencing grade trypsin; $250 \mu \mathrm{L}$ of this solution were added to each of the digest samples from overnight, and the reactions were left at $37^{\circ} \mathrm{C}$ for 4 hours with shaking (600 rpm).
Thirty-five $\mu \mathrm{L} 10 \%$ formic acid were added to the $500 \mu \mathrm{L}$ digest sample $(0.7 \%$ final concentration of formic acid) to stop the digestion. The digested solution was diluted in 7.5 $\mathrm{mL}$ of acetonitrile containing $0.3 \%$ formic acid.

\section{HILIC solid phase extraction (SPE) of peptides}

PolyhydroxyethylA SPE $12 \mu \mathrm{m}, 300 \mathrm{~A}, 300 \mathrm{mg}$ cartridges (obtained from PolyLC) were used for the HILIC procedure. Prior to use, cartridges required an overnight soak in $50 \mathrm{mM}$ formic acid followed by rinsing with water the following day. Cartridges were preconditioned with $2 \mathrm{~mL}$ of Buffer A $(90 \%$ acetonitrile, $5 \mathrm{mM}$ ammonium formate, $\mathrm{pH} 2.7$ ) followed by $2 \mathrm{~mL}$ of Buffer B (5mM ammonium formate, $\mathrm{pH} 2.7)$ and finally re-equilibrated with $10 \mathrm{~mL}$ Buffer $\mathrm{A}$. The diluted samples were loaded onto the cartridges and washed with a further $10 \mathrm{~mL}$ Buffer A. Finally, peptides were eluted in $1 \mathrm{~mL}$ Buffer C (9 parts Buffer B plus 1 part Buffer A) and the samples dried on a Speedvac to remove organic solvent prior to LC-MS/MS analysis.

\section{LC-MS/MS analysis}

Lyophilised digests were resuspended in $50 \mu \mathrm{L}$ of $0.1 \%$ TFA to give an approximate concentration of $1 \mu \mathrm{g} / \mu \mathrm{L}$. One $\mu \mathrm{L}$ injection volumes were used throughout resulting in an on-column peptide loading of approximately $1 \mu \mathrm{g}$ per injection. Analysis was performed in quintuplicate for each sample. All LC-MS/MS analyses were performed on an LTQ Orbitrap XL mass spectrometer coupled to an Ultimate 3000 RSLCnano system (Thermo Scientific). One $\mu \mathrm{L}$ injection volumes were used throughout and samples loaded directly onto the analytical column, PepMap RSLC C18, $2 \mu \mathrm{m} \times 75$ $\mu \mathrm{m}$ id $\times 50 \mathrm{~cm}$ (Thermo Scientific). The composition (v/v) of LC buffers were as follows; Buffer A - 99.9\% water plus $0.1 \%$ formic acid and Buffer B $-80 \%$ acetonitrile, $19.9 \%$ water and $0.1 \%$ formic acid. Peptides were loaded directly onto the column at a flow rate of $400 \mathrm{nl} / \mathrm{min}$ with an initial mobile phase composition of $1 \% \mathrm{~B}$. The organic strength was increased linearly from $1 \%$ to $22.5 \%$ B over 22.5 minutes again at $400 \mathrm{nl} / \mathrm{min}$, followed by an increase to $24.8 \% \mathrm{~B}$ over the next 2.6 minutes with a concomitant reduction in flow rate to $300 \mathrm{nl} / \mathrm{min}$, and to $39 \% \mathrm{~B}$ over a further 14 minutes. A further increase to $60 \% \mathrm{~B}$ over the next 5 minutes was followed by a ramp to $95 \%$ B over 2.5 minutes where it was held for a further 2 minutes. The column was then allowed to re-equilibrate to $1 \% \mathrm{~B}$ for a total analysis time of 74 minutes. The mass spectrometer was instructed to perform data dependent acquisition on the top six precursor ions, which were measured in the Orbitrap FTMS detector over the mass range 370-1200 $\mathrm{m} / \mathrm{z}$, at a nominal resolution of 60,000 . MS/MS spectra were acquired in the ion trap under CID conditions with normalized collision energy of 35 , isolation width of 3 Th, Q value of 0.25 and $30 \mathrm{~ms}$ activation time. Gas-phase 
fractionation was performed on the five replicate injections such that MS/MS data was collected for precursor ion range $370-494 \mathrm{~m} / \mathrm{z}$ Injection $1,494-595 \mathrm{~m} / \mathrm{z}$ Injection 2, 595-685 m/z Injection 3, 685-817 m/z Injection 4 and $817-1200 \mathrm{~m} / \mathrm{z}$ Injection 5.

\section{Analysis}

Xcalibur raw data files acquired on the LTQOrbitrap XL were directly imported into Progenesis LCMS software (Waters Corp) for peak detection and alignment. Data were analysed using the Mascot search engine. Five replicates were analysed for each sample type $(N=5)$.

\section{Ingenuity pathway analyses}

Pathway and function analyses were generated using Ingenuity Pathway Analysis (IPA) (Ingenuity systems, http://www.ingenuity.com), which assists with proteomics data interpretation via grouping differentially expressed genes or proteins into known functions and pathways. Pathways with a $z$ score $>1.9$ were considered as significantly activated, and pathways with a $z$ score $<-1.9$ were considered as significantly inhibited.

\section{Statistical analyses}

ANOVA was used for statistical comparison of three or more groups. For normalised data, one-sample $t$ test was performed. All data are reported as mean \pm standard deviation of the mean (SEM). All experiments were performed at least three times with reproducible results unless otherwise stated. $P$ values lower than 0.05 were considered significant $\left({ }^{*} P<0.05,{ }^{* *} P<0.01,{ }^{* * *} P<0.001\right)$. Microsoft Excel was used to produce all graphs except FACS analysis graphs, in which case FlowJo was used.

\section{Author contributions}

Professors Lisanti and Sotgia conceived and initiated this project. All the experiments described in this paper were performed by Dr. Maria Peiris-Pagès, who then generated the final figures and tables for the paper. Dr. Gloria Bonuccelli performed the experiments on tumoursphere formation. Dr. Maria Peiris-Pagès wrote the first draft of the manuscript, which was then further edited by all the other co-authors.

\section{ACKNOWLEDGMENTS}

We are grateful to the University of Manchester that allocated start-up funds and administered a donation, which provided all the necessary resources required to start and complete this drug discovery project (to M.P.L. and F.S.).

Currently, the Sotgia and Lisanti Laboratories are supported by private donations, the British Schools and
Universities Foundation, the Healthy Life Foundation (HLF) and the Foxpoint Foundation.

\section{CONFLICTS OF INTEREST}

MPL and FS hold a minority interest in Lunella, Inc.

\section{REFERENCES}

1. Vyas S, Zaganjor E, Haigis MC. Mitochondria and Cancer. Cell. 2016; 166:555-566.

2. Mishra P, Chan DC. Metabolic regulation of mitochondrial dynamics. J Cell Biol. 2016; 212:379-387.

3. Senft D, Ronai ZA. Regulators of mitochondrial dynamics in cancer. Curr Opin Cell Biol. 2016; 39:43-52.

4. Rosdah AA, K Holien J, Delbridge LM, Dusting GJ, Lim SY. Mitochondrial fission - a drug target for cytoprotection or cytodestruction? Pharmacol Res Perspect. 2016; 4:e00235.

5. Cassidy-Stone A, Chipuk JE, Ingerman E, Song C, Yoo C, Kuwana T, Kurth MJ, Shaw JT, Hinshaw JE, Green DR, Nunnari J. Chemical inhibition of the mitochondrial division dynamin reveals its role in Bax/Bak-dependent mitochondrial outer membrane permeabilization. Dev Cell. 2008; 14:193-204.

6. Kong B, Tsuyoshi H, Orisaka M, Shieh DB, Yoshida Y, Tsang BK. Mitochondrial dynamics regulating chemoresistance in gynecological cancers. Ann N Y Acad Sci. 2015; 1350:1-16.

7. Xie Q, Wu Q, Horbinski CM, Flavahan WA, Yang K, Zhou W, Dombrowski SM, Huang Z, Fang X, Shi Y, Ferguson AN, Kashatus DF, Bao S, et al. Mitochondrial control by DRP1 in brain tumor initiating cells. Nat Neurosci. 2015; 18:501-510.

8. Katajisto P, Dohla J, Chaffer CL, Pentinmikko N, Marjanovic N, Iqbal S, Zoncu R, Chen W, Weinberg RA, Sabatini DM. Stem cells. Asymmetric apportioning of aged mitochondria between daughter cells is required for stemness. Science. 2015; 348:340-343.

9. Prieto J, Leon M, Ponsoda X, Sendra R, Bort R, FerrerLorente R, Raya A, Lopez-Garcia C, Torres J. Early ERK1/2 activation promotes DRP1-dependent mitochondrial fission necessary for cell reprogramming. Nat Commun. 2016; 7:11124.

10. Vazquez-Martin A, Cufi S, Corominas-Faja B, OliverasFerraros C, Vellon L, Menendez JA. Mitochondrial fusion by pharmacological manipulation impedes somatic cell reprogramming to pluripotency: new insight into the role of mitophagy in cell stemness. Aging (Albany NY). 2012; 4:393-401. https://doi.org/10.18632/aging. 100465.

11. Fiorillo M, Lamb R, Tanowitz HB, Cappello AR, MartinezOutschoorn UE, Sotgia F, Lisanti MP. Bedaquiline, an FDA-approved antibiotic, inhibits mitochondrial function and potently blocks the proliferative expansion of stem-like cancer cells (CSCs). Aging (Albany NY). 2016; 8:15931607. https://doi.org/10.18632/aging. 100983. 
12. Fiorillo M, Lamb R, Tanowitz HB, Mutti L, KrsticDemonacos M, Cappello AR, Martinez-Outschoorn UE, Sotgia F, Lisanti MP. Repurposing atovaquone: targeting mitochondrial complex III and OXPHOS to eradicate cancer stem cells. Oncotarget. 2016; 7:34084-34099. https://doi.org/10.18632/oncotarget.9122.

13. Lamb R, Ozsvari B, Lisanti CL, Tanowitz HB, Howell A, Martinez-Outschoorn UE, Sotgia F, Lisanti MP. Antibiotics that target mitochondria effectively eradicate cancer stem cells, across multiple tumor types: treating cancer like an infectious disease. Oncotarget. 2015; 6:4569-4584. https:// doi.org/10.18632/oncotarget.3174.

14. Cai J, Wang J, Huang Y, Wu H, Xia T, Xiao J, Chen X, Li H, Qiu Y, Wang Y, Zhang B, Wu H, Zhang Y, et al. ERK/ Drp1-dependent mitochondrial fission is involved in the MSC-induced drug resistance of T-cell acute lymphoblastic leukemia cells. Cell Death Dis. 2016; 7:e2459. https://doi. org/10.18632/aging.100808.

15. Peiris-Pages M, Smith DL, Gyorffy B, Sotgia F, Lisanti MP. Proteomic identification of prognostic tumour biomarkers, using chemotherapy-induced cancer-associated fibroblasts. Aging (Albany NY). 2015; 7:816-838.

16. Yue PY, Leung EP, Mak NK, Wong RN. A simplified method for quantifying cell migration/wound healing in 96-well plates. J Biomol Screen. 2010; 15:427-433.

17. Teslaa T, Teitell MA. Pluripotent stem cell energy metabolism: an update. EMBO J . 2015; 34:138-153.

18. Liu S, Cong Y, Wang D, Sun Y, Deng L, Liu Y, MartinTrevino R, Shang L, McDermott SP, Landis MD, Hong $\mathrm{S}$, Adams A, D'Angelo R, et al. Breast cancer stem cells transition between epithelial and mesenchymal states reflective of their normal counterparts. Stem Cell Reports. 2014; 2:78-91.

19. Mitra K. Mitochondrial fission-fusion as an emerging key regulator of cell proliferation and differentiation. Bioessays. 2013; 35:955-964.

20. Qian W, Wang J, Roginskaya V, McDermott LA, Edwards RP, Stolz DB, Llambi F, Green DR, Van Houten B. Novel combination of mitochondrial division inhibitor 1 (mdivi1) and platinum agents produces synergistic pro-apoptotic effect in drug resistant tumor cells. Oncotarget. 2014; 5:4180-4194. https://doi.org/10.18632/oncotarget.1944.

21. Schatton T, Frank MH. The in vitro spheroid melanoma cell culture assay: cues on tumor initiation? J Invest Dermatol. 2010; 130:1769-1771.

22. Zhao J, Zhang J, Yu M, Xie Y, Huang Y, Wolff DW, Abel PW, Tu Y. Mitochondrial dynamics regulates migration and invasion of breast cancer cells. Oncogene. 2013; 32:4814-4824.

23. Caino MC, Altieri DC. Cancer cells exploit adaptive mitochondrial dynamics to increase tumor cell invasion. Cell Cycle. 2015; 14:3242-3247.

24. Gugnoni M, Sancisi V, Gandolfi G, Manzotti G, Ragazzi M, Giordano D, Tamagnini I, Tigano M, Frasoldati A, Piana S, Ciarrocchi A. Cadherin-6 promotes EMT and cancer metastasis by restraining autophagy. Oncogene. 2017; 36:667-677.

25. Martinez-Outschoorn UE, Peiris-Pages M, Pestell RG, Sotgia F, Lisanti MP. Cancer metabolism: a therapeutic perspective. Nat Rev Clin Oncol. 2017; 14:113.

26. Peiris-Pages M, Martinez-Outschoorn UE, Pestell RG, Sotgia F, Lisanti MP. Cancer stem cell metabolism. Breast Cancer Res. 2016; 18:55.

27. Shaw FL, Harrison H, Spence K, Ablett MP, Simoes BM, Farnie G, Clarke RB. A detailed mammosphere assay protocol for the quantification of breast stem cell activity. $\mathrm{J}$ Mammary Gland Biol Neoplasia. 2012; 17:111-117.

28. Peiris-Pages M, Sotgia F, Lisanti MP. Chemotherapy induces the cancer-associated fibroblast phenotype, activating paracrine Hedgehog-GLI signalling in breast cancer cells. Oncotarget. 2015; 6:10728-10745. https://doi. org/10.18632/oncotarget.3828. 